LBL-36467

UC- -314

\title{
Combined Analysis of Surface Reflection Imaging and Vertical Seismic Profiling at Yucca Mountain, Nevada
}

\author{
T.M. Daley, E.L. Majer, and E. Karageorgi \\ Earth Sciences Division \\ Lawrence Berkeley Laboratory \\ University of California \\ Berkeley, California 94720
}

August 1994

This work was supported by the U.S. Department of Energy Contract No. DE-AC03-76SF00098 for the Director, Office of Civilian Radioactive Waste Management, Office of Geologic Disposal, and was administered by the Nevada Operations Office, U.S. Department of Energy, in cooperation with the U.S. Geological Survey, Denver. 


\section{DISCLAIMER}

Portions of this document may be illegible in electronic image products. Images are produced from the best available original document. 


\section{Introduction}

This report presents results from surface and borehole seismic profiling performed by the Lawrence Berkeley Laboratory (LBL) on Yucca Mountain. This work was performed as part of the site characterization effort for the potential high-level nuclear waste repository. Our objective was to provide seismic imaging from the near surface ( 200 to $300 \mathrm{ft}$. depth) to the repository horizon and below, if possible. Among the issues addressed by this seismic imaging work are location and depth of fracturing and faulting, geologic identification of reflecting horizons, and spatial continuity of reflecting horizons. We believe our results are generally positive, with some specific successes. This was the first attempt at this scale using modern seismic imaging techniques to determine geologic features on Yucca Mountain. We recognize that we still have work to do to fully optimize the data acquisition and processing. Since previous seismic reflection studies in the area have had mixed results with difficulty imaging coherent reflections (e.g. Harding, 1988, McGovern, et al., 1983), we feel the data presented here is part of the learning process for optimal application of seismic imaging methods on the Yucca Mountain site.

The principle purpose of this report is to present the interpretation of the seismic reflection section in a geologic context. We do not intend to report on complete details of the processing steps, sequences and techniques used. Those processing results were presented earlier in Majer and Karageorgi, 1993, and Daley and Majer, 1993. It should be noted that the lithologic interpretations presented in this report are only intended to be approximate and a best guess by a seismic interpreter which may differ from the accepted lithostratigraphic model mapped by geologists. The model derived from seismic results will not be the same as that derived from other techniques, but it is intended to aid progress toward a single 3-D model of Yucca Mountain.

Three surface reflection profiles were acquired and processed as part of this study. Because of environmental concerns, all three lines were on preexisting roads. The locations of the profile lines are shown in Fig 1a. Figs $1 \mathrm{~b}-1 \mathrm{~d}$ show geologic cross-sections along the azimuth of each line; these figures were generated using the computer-assisted lithostratigraphic model being compiled by the U.S. Geological Survey (USGS) (USGS Rock Characteristics Section, written communication, 1994). Line 1 crossed the mapped surface trace of the Ghost Dance fault and it was intended to study the dip and depth extent of the fault system. Vertical seismic profile (VSP) surveys were acquired in and around well WT-2 (which is located along surface line 1) as part of the Ghost Dance fault imaging. . Line 2 was acquired along Drill Hole wash. VSP surveys were acquired in well NRG-6 which is located next to line 2. The Drill Hole wash studies were intended to help the ESF north ramp design activities. Line 3 was acquired along Yucca Crest. No VSP studies were performed along line 3. Line 3 was designed to image geologic horizons which were thought to be less faulted along the ridge. Unfortunately, line 3 proved to have poor data quality, in part because of winds, poor field conditions and limited time. Because of this, Line 3 was not interpreted as part of this report although we do provide a stacked common depth point (CDP) section for Line 3. Our processing and interpretation efforts were focused on lines 1 and 2 and their associated VSP studies.

\section{Background}

Geophysical characterization of the subsurface using seismic methods has three basic types of data acquisition, surface to surface (surface reflection or refraction profiling), vertical seismic profiling (VSP) and cross-hole seismic profiling. Before acquiring the surface seismic lines, we had álready carried out VSP at WT-2 and NRG-6, and cross-hole imaging at the C-hole complex. 
The VSP data indicated reflecting horizons, thus it was logical from this evidence that a surface reflection profile may yield useful information over a wider volume than VSP provides. In addition, LBL had just completed a high resolution surface reflection line on Rainier Mesa in area 12 at the Nevada Test Site, which showed encouraging results using modern state-of-the-art equipment for surface seismic reflection profiling in order to image the tuffs (Majer, et. al., 1994). Therefore, three seismic reflection profiles were acquired during the week of October 25, 1993 in the region of the WT-2 VSP (line Yucca-1, or Line-1, starting east of UZ-16 and running up the road past WT-2), the NRG-6 VSP (line Yucca-2, or Line-2, starting at NRG-7 and running down the road in Drill Hole Wash past NRG-6 to the edge of the corporation yard) and a line along the crest of Yucca Mountain starting at UZ-6 and extending approximately 3000 feet north along the crest road towards H-5 (line Yucca-3, or Line -3). As in the case of the VSP work the principal reason for the work was to image from the near surface to at least the repository horizon and below if possible. The objective was to provide information on faulting/fracturing and structure/lithology to provide input to ESF design activities. In addition these data would also provide information on geologic structure for input and control of the 3-D site rock characteristics model.

\section{VSP Data Acquisition}

Because the VSP data were used to interpret and guide the processing of the reflection data, we provide a short description of the VSP work at WT-2 and NRG-6. Vertical Seismic Profile (VSP) surveys were obtained in boreholes NRG-6 and WT-2 on Yucca Mountain during August 1993. Our VSP work began in borehole NRG-6 which is approximately 1100 feet deep. We performed three complete VSP surveys in NRG-6 between August 23 and 25. Our initial survey had P- and S-wave vibroseis sources on the well pad, giving a measurement of rock velocities for vertically propagating waves ("zero offset"). In all NRG-6 offsets we recorded data at 20 foot intervals from 1080 feet to 40 feet from both $\mathrm{P}$ - and S-wave sources giving 53 3-component recordings for each source. Both sources used a 12 second sweep from 10 to $82 \mathrm{~Hz}$. A total of 16 seconds of data were recorded, giving 4 seconds of "listen time" after each sweep. Two sweeps were recorded for each source at each depth to help cancel background noise. The second two surveys had source locations 90 degrees apart and offset from the well pad. These two VSP's used the same source sweeps and depth sampling as the initial survey at the NRG-6 well pad. Offset number 2 source point was 377 feet from the well and offset number 3 source point was 679 feet from the well, with respective azimuths of 80 and 170 degrees east of north, measured from the well head. By having source locations separated by 90 degrees, we wanted to measure the anisotropy of the rock as a function of depth in the well. The source offset allows us to obtain attenuation information for the rock between the source and well.

The VSP work in well WT-2 was performed from August 25 to 27, 1993. Well WT-2 is 2060 feet deep. We acquired 2 complete VSP surveys (near offset at 80 feet and a far offset at 1000 feet) at levels from 2010 feet to 100 feet using 30 foot depth intervals giving 63 3component recordings. In addition, we performed a single depth (1500 feet), multiple source walkaway VSP survey with 21 recordings at 50 foot offset intervals east from the well along the road from UZ-16 to WT-2, i.e. on the same road that the surface reflection profile was carried out, crossing the mapped location of the Ghost Dance fault. The near offset VSP survey at WT-2 used the sources on the well pad for vertical wave propagation. Both P-and S-wave sources used a 12 second, 10 to $82 \mathrm{~Hz}$ sweep with 16 seconds total recording time. This survey provided P- and Swave velocity information to 2010 feet, allowing calculation of Poisson's ratio and other seismic attributes over this depth range. The far offset VSP survey at WT-2 had sources at a 1000 foot 
offset, across the surface trace of the Ghost Dance fault. The sweep parameters and depth sampling were the same as the initial survey. Because of the larger offset, we recorded up to 9 individual sweeps for each source-receiver pair in order to improve signal-to-noise ratios. The offset distance used in the second survey allowed us to measure the seismic properties for the rock between the well and the source location. The objective is to identify variation in rock fracturing, or other rock properties between the source location and the well. The walkaway survey was performed with the receiver at 1500 feet. Data was recorded using the S-wave source at 50 foot intervals from an offset of 1050 feet (the same location as survey \#2) to an offset of 50 feet. This walkaway VSP was designed to try and locate the Ghost Dance fault zone. We expected the fault to have anomalous velocity and attenuation properties, which could be identified and located with 50 to 100 foot accuracy within the walkaway data set.

\section{Surface Reflection Acquisition}

As stated above three high resolution, surface reflection lines were carried out at Yucca Mountain. The approximate locations of the lines are shown on Figure 1. Lines 2 and 3 are relatively straight, whereas Line 1 is relatively crooked. Due to environmental concerns the lines were restricted to existing roads. Line 1 was the longest, approximately $6500 \mathrm{feet}$, Line 2 was approximately 5200 feet, and Line 3 was approximately 3000 feet long. More precise coordinates of the lines and some reference points are given in Nevada state plane coordinates in Table 1 . The G4 and G5 locations in Table 1 are gravity stations along a gravity line run parallel to Line 1, and can be used for reference. Each geophone location along the seismic reflection lines was surveyed by conventional optical survey methods to within 0.1 foot. The end and middle values along the lines were checked with a Global Positioning Survey (GPS). The values agreed to within 0.1 foot, therefore, we have a high degree of confidence in the survey coordinates. The lines were chosen in three different geologic conditions, perpendicular to major faulting (Line 1, across the southern structure of the Ghost Dance fault (GDF)), almost parallel to major faulting (Line 2, along Drill Hole Wash ), and in an area of assumed relative sparse faulting (Line 3, along the crest of Yucca Mountain).

At the beginning of the survey noise tests were carried out along Line 1 to determine the frequency range that could be obtained and the optimal source and receiver spacing. This resulted in 1 second of data with a sampling rate of 2000 samples/seconds using a Bison Model 90-48 (48 channel, 24 bit digital recorder) with a bandpass filter of 64 to 250 Hertz. The source of energy was a Bison EW-2 impact source with a rating of 30,000 pounds of force. Single Mark product $30 \mathrm{Hertz}$ geophones were placed at 40 foot intervals starting at station 101 on Line 1, and at station

110 on Line 2 . On Line 3 the geophone spacings were at 20 foot intervals. A source array was used to reduce ground roll. In general four sets of two shots spaced every 10 feet were used as a source array, resulting in a stack of eight shots per source point. The shotpoint interval was $40 \mathrm{ft}$. The nominal CDP fold was 24. Each stack of eight shots was played back in the field for data quality. The geometry of the shooting was to "push" the 48 sensor array from the first station through the end of the line, i.e., we used an off end array to minimize ground roll interference in the first several hundred milliseconds of data, thereby enhancing the shallow structure. The gap between the source and the first geophone was $20 \mathrm{ft}$.

\section{Processing Sequence, Line 1 and 2}

Line 1 consists of 153 shots and Line 2 consists of 99 shots. Depending on the processing 
needs of the data we used both SierraSeis version 5.1 and ProMAX version 40.29 seismic software. Initial processing was reported in Majer and Karageorgi (1993). Shot gathers using the raw field data did not reveal any reflections over the entire shot gather, but only over part of any one shot gather, presumably because of heterogeneous structure. Further processing was applied to the shot gather data, which included trace editing and muting, band-pass filtering to eliminate higher frequencies (above $150 \mathrm{~Hz}$ ), time-variant spectral whitening, automatic gain control (AGC) and lateral scaling across the traces in the gather, muting (different for each line), and finally, filtering in the frequency-wave number domain $(\mathrm{F}-\mathrm{K})$, which eliminates noise based on its slope. Careful use of F-K filtering was applied so that only "down-going" energy was removed from the shot gathers. The F-K filtering considerably improved the data quality, however, edge effects from the Fourier transformation (FFT) contaminated the gathers. For this reason, many F-K filtering test runs were performed before we decided on final parameters which minimized the FFT edge effects. This processing sequence revealed reflections in the shot gathers, and consequently in the CDP stacked profiles. A crooked line geometry was designed for CDP binning of line 1 . We generated constant velocity CDP stacks of Line 1 with stacking velocities from $6000 \mathrm{ft} . / \mathrm{sec}$ to $16000 \mathrm{ft} . / \mathrm{sec}$ at intervals of $1000 \mathrm{ft} . / \mathrm{sec}$. A $12,000 \mathrm{ft}$./s stacking velocity was chosen for Line 1 . Elevation statics, residual statics and surface consistent statics were applied. Post-stack processing of the CDP section consisted of an F-X (frequency-offset) Weiner-Levinson deconvolution which reduces spatially random noise, F-K filtering to reduce steeply dipping artifacts, time-depth conversion using VSP measured velocities and AGC. It is important to note that F-K filtering was "lightly" applied, and only used to reduce the effects of crosscutting events that were artifacts of processing. F-K filtering was not used to increase the coherence of horizontal events, but only used to remove events that were obscuring horizontal or close to horizontal reflectors. It is important to note that no coherency filtering was used to enhance any reflections.

The lack of strong coherent reflectors in many gathers prevented us from developing a 2-D velocity model and from utilizing more advanced imaging techniques such as pre- or post-stack migrations. Other types of attribute analysis were attempted on the final sections, such as instantaneous frequency, Hilbert transform envelopes, and instantaneous phase, but none of these proved useful for interpreting the sections.

Similar processing steps were followed for Line 2, with the exception of the application of predictive deconvolution with a $250 \mathrm{~ms}$ gate length. The improvement of the data after deconvolution is debatable. The field data quality of Line 2 is considerably better than that of Line 1. We used the F-K filtered shot gathers for the generation of the constant velocity stacks for consistency with Line 1 . A stacking velocity of $13,000 \mathrm{ft} / \mathrm{s}$ was chosen for line 2 .

\section{Interpretation of Line 1 - Ghost Dance Fault Study}

The $6500 \mathrm{ft}$. line 1 data was acquired and processed as described above. The line is located as shown in Figs. 1a and 1b. The uninterpreted CDP profile for line 1 is shown in Fig 2 . All the following interpretations were done on this depth section which includes the entire 1 second of recorded data. However, please note that the time to depth conversion is not completely accurate because no wells penetrate the entire $1 \mathrm{sec}$ of data and only two wells (WT-2 and UZ-16) are on the surface line. The time to depth conversion used average velocities measured by VSP in WT-2 (velocities listed in Daley and Majer, 1993). We believe the depth accuracy should be within $+/-$ $5 \%$ near WT-2 and no worse than $+/-10 \%$ anywhere on the section.

Our interpretation effort began with identification of important reflectors. Fig 3 shows line 
1 with notable reflections enhanced for easier identification. These are reflections which are identifiable from VSP or those which are most spatially continuous across the section. For example, the WT-2 VSP showed a major reflection at 1100 to 1200 feet. We interpret this reflection to be associated with the vitrophyre at the base of the Topopah Spring formation. Having identified this reflection, we attempt to follow it across the section to UZ-16 where we again know the depth. However there are discontinuities between the wells which make continuous reflector identification difficult. The reflection section is largely characterized by short segments of discontinuous reflections rather than long continuous reflections. This lack of continulity does add ambiguity to our interpretation.

Reflections below the wells were identified by signal strength (relative amplitude), spatial continuity (adjacent traces which had the same event) and wavelet characteristics (visual identification of phase and frequency content variations). These identifications were interpretive decisions, not analytic determinations. Fig 4 is an interpretation showing identified reflectors highlighted in red.

The next step in the interpretation was to identify lithologic boundaries based on available well information, and place these lithologic boundaries on the seismic section, as shown in Fig 5. The information used to identify the lithology in Fig 5 came from wells WT-2, UZ-16 and H-4 (see Fig. 1a and b for locations) (Gelsin, et. al., in press, Nelson, et. al., 1991). The lithologic boundaries shown in Fig 5 were interpolated as straight lines between the boundaries observed in these wells. The next step in our analysis took this lithologic interpretation and modified it to match the seismic response; this result is shown in Fig 6. The lithology shown in Fig 6 (and Figs $7 a$ and $b$ ) is an interpretive model based on seismic reflectors. The reflectors were identified as likely lithologic contacts where they intersect the wells. We then attempted to follow the reflector across the section and maintain the lithologic identification. The well information from $\mathrm{H}-4$ was used to identify reflectors below WT-2 and UZ-16. Please note that our lithologic coloration of the seismic section is a visual guide and the color boundaries are not meant to be an accurate location of lithologic boundaries. Also note that the coloration of the Topopah Spring formation (yellow) on Line 1 may include some parts of the Tiva Canyon formation.

At this point it is possible to give lithologic interpretation to the major reflections. This interpretation was done with the aid of available well logs in WT-2, H-4 and UZ-16 in addition to geologic interpretations by R. Spengler (personnel communication, 1994). Beginning at the top of the section our interpretation of the approximate depth and lithologies of the notable reflections are as follows:

1) The transition between the upper lithophysal zone and the middle non-lithophysal zone of the Topopah Spring Tuff at about 500 feet.

2) The densely welded vitrophyre at the base of the Topopah Spring Tuff at about 1200 feet.

3) Top of the moderate to densely welded zone of the Prow Pass Tuff at about 1500 feet.

4) Top of the densely welded zone of the Bullfrog Tuff at about 2000 feet.

5) Top of the densely welded zone at the top of the Tram Tuff at about 2500 to 3000 feet.

6) Base of the densely welded zone at the top of the Tram Tuff at about 3900 feet.

Finally, we present two different interpretations for the presence of faulting on Line 1 (Figs. $7 \mathrm{a}$ and $7 \mathrm{~b}$ ). Please note that the nature of the reflections in line 1 is fairly short length (at most 500 to 1000 feet) with numerous discontinuities: With the numerous discontinuities on this section, it is difficult to uniquely locate faults and their dip.

Our interpretation of faulting was guided by current estimates of geologic structure. While discontinuities in reflectors could be caused by faulting or by strong lateral variation in rock properties, we do have several observations which indicate a fault controlled structural model. We 
can identify reflectors in the offset VSP which tie to the surface data with the same discontinuity, and we observe vertical features in the walkaway VSP. We observe the same discontinuity offset (e.g.. down-thrown to the west) for different reflectors at different depths under the same surface location. We observe that reflector continuity increases with depth; this observation can imply vertical features since they will scatter more energy shallow where the aperture of the geophone spread is poor (long surface line vs. shallow depths). The vertical displacement of reflectors and their offset down to the west is consistent with other observations on and around Yucca Mountain. We believe these observations justify a fault controlled model. However, the exact nature of the faulting is more difficult to identify. We feel different fault models can be interpreted on Line 1 and we have provided two such models. Fig. $7 \mathrm{a}$ is a listric type fault interpretation, Fig. $7 \mathrm{~b}$ is a planer fault model. The identification of fault location in the subsurface depends on the fault model one chooses. Because of the large number of reflector discontinuities, it is difficult to accurately constrain fault dips. We have attempted to use previous geologic studies which place steeply west dipping (80 to 90 degrees) faults in the region of line 1 (Scott and Bonk, 1984, Spengler and Fox, Jr., 1989).

Despite the generally discontinuous nature of the seismic reflectors, we do observe some relative increase in reflector continuity with increasing depth. This increase in reflector continuity with depth also implies that the lithologic units at depth may be better seismic reflectors, implying a general change in lithologic properties as a function of depth. In this context, it is worthwhile to note the lithophysal zones only occur in the Tiva Canyon and Topopah Spring Tuffs ( above the Calico Hills Formation and the Crater Flat Group). These lithophysal zones are also in the unsaturated zone, and the seismic response is affected by saturation. We can not yet state the degree to which saturation and the static water level (about $1875 \mathrm{ft}$. in WT-2) affects the reflectivity. The seismic reflectivity of the deeper formations may also be affected by the presence of interstratification of zeolitized nonwelded and high-temperature crystallized rocks in the Prow Pass, Bullfrog, and Tram Tuffs.

\section{Interpretation of WT-2 VSP}

The acquisition and processing of the VSP data is described above and in detail in Daley and Majer, 1993. The main interpretation uses of the WT-2 VSP are as follows:

1) Velocities measured by the zero offset VSP are used for processing and depth-conversion of the surface profile.

2) Derivation of a reflection seismogram from the zero offset reflected arrivals gives accurate depth of reflections within the well and it can tie reflections seen on surface profiles to true depth of lithology.

3) Offset CDP mapping of the 1000 foot offset $P$ and S-wave data. This VSP-CDP transform gives a CDP type profile of reflections between the well and (halfway to) the source offset location (Balch and Lee, 1984).

4) Walkaway VSP (where the sensor is fixed at depth and the source is placed at equal intervals along a surface line). This survey is analyzed for reflections from near-vertical reflectors (e.g. fault zones) and for variation in travel time along the source line.

The zero-offset velocity analysis, including $\mathrm{P}$ and $\mathrm{S}$-wave travel times, interval and average velocities, and Poisson's Ratio, was reported in Daley and Majer, 1993. The average P-wave velocities were used to guide the time to depth conversion of surface Line 1.

The P-wave reflection seismogram for WT-2 is shown in Fig 8 a. Since the frequency content of the VSP survey was lower than the surface seismic survey, we provide a high-pass 
filtered version of the reflection seismogram in Fig $8 \mathrm{~b}$. These VSP reflection seismograms can be overlaid on line 1 to compare reflection character and depth. Major reflections (those which appear on both line 1 and the VSP) are seen at about 550, 1100, 1400, 1700 and 2400 feet.

The VSP-CDP mapping of offset 1 (1000 feet east of WT-2) P-wave source is shown in Figs $9 a$ and $b$ (two scales 1:6000 for overlay on line 1 and a $2 x$ horizontal exaggeration for better observation of reflections). The S-wave source VSP-CDP transform, using a horizontal geophone component, is shown in Figs $10 \mathrm{a}$ and $\mathrm{b}$. We see a possible tie to reflections observed in the surface line, including offset of the 1100 foot reflector which steps down (from about $900 \mathrm{ft}$. to about $1100 \mathrm{ft}$ ) to the west. While there are some reflectors which do tie, the reflectivity observed in the VSP data is not entirely matched in line 1 . Both $P$ and $S$ VSP-CDP sections show apparent dipping reflections between 1500 and 2500 feet. We believe these may be associated with the Ghost Dance fault system, however the dips appear too shallow to be correctly imaging this fault system and may be diffractions.

Initial, analysis of the walkaway VSP survey looked at the variation of travel time along the walkaway (covering 1000 feet east of WT-2). Fig 11 shows there is a large change in travel time between 550 and 600 feet. This is probably due to crossing a fault controlled feature. The walkaway VSP survey was also processed in a VSP-CDP type manner, but, with the geometry of a walkaway survey, a VSP-CDP section can show reflections from near vertical reflectors. In this case the source and receiver locations are reversed from a typical VSP-CDP transform and the subsurface coverage is rotated 90 degrees (i.e. coverage is halfway down to the sensor instead of halfway away from the well). A VSP-CDP mapping velocity of $3500 \mathrm{ft} . / \mathrm{s}$ provided the best vertical events, implying S-wave reflections are the dominant energy (the source was S-wave). This walkaway-CDP plot is shown in Fig 12 at the same scale as line 1 for overlay. We see three reflections within the $1000 \mathrm{ft}$. offset and $750 \mathrm{ft}$. depth region imaged by the walkaway-CDP. These reflections are nearly coincident with the three faults interpreted east of WT-2 on line 1 . We believe the most likely cause of these near-vertical reflections are interfaces associated with the Ghost Dance fault system. We do not know if these are discrete faults or zones of lithologic alteration associated with faulting. The variation in dip observed in these reflections (Fig. 12) is quite sensitive to velocity and should not be interpreted. By using the surface data to project faults downward (Fig 7a, 7b) it appears plausible that the Ghost Dance fault system may intersect WT-2 near the well bottom, although previous well studies (including borehole televiewer) did not observe the fault (Spengler, personnel communication, 1994 ).

\section{Interpretation of Line 2 - Drill Hole Wash Study}

The $4000 \mathrm{ft}$. line of data acquired in Drill Hole Wash was acquired and processed as described above. Figure 13 shows the surface geology and VSP locations in Drill Hole Wash. Line 2 is located between wells A-1 and NRG-7 (see also Fig 1a and 1c). The uninterpreted CDP depth section is shown in Fig 14. All interpretation were done on this depth section which includes the full 1 second of data. No further processing was performed for the interpreted figures. As with line 1, the time to depth conversion was done with VSP data (from NRG-6 for line 2) and these depths should be considered approximate because the well is only 1100 feet deep. We believe the depth accuracy should be within $+/-5 \%$ near NRG- 6 and no worse than $+/-10 \%$ anywhere on the section. A section with highlighted reflections is shown in Fig 15. These reflections were identified using the same criteria described above for line 1 . Fig 16 shows the lithology as identified from nearby wells ( A-1 and H-1, see Fig. 1a) (lithology from USGS Rock Characteristics Section, written communication, 1994) and linearly interpolated across the seismic 
section. Fig 17 shows the lithology adjusted to match the interpreted seismic data. The lithology in Fig. 17 may disagree with some well data, but it is intended to give a model based on seismic data. As with line 1 , please note that the colored lithology is intended as a visual aid and is not intended to accurately represent the location of lithologic boundaries. The major reflectors highlighted are interpreted to be associated with the following lithologic features (lithology from Gelsin and Moyer, 1994, Gelsin, et. al., in press, and USGS Rock Characteristics Section, written communication, 1994; listed depths vary across section):

1) The vitric zone at the top of the Topopah Spring Tuff at about 250 feet.

2) The top of the upper lithophysal zone of the Topopah Spring Tuff at about 480 feet or the increasing lithophysae at $520 \mathrm{ft}$.

3) The top of the lower lithophysal zone of the Topopah Spring Tuff at about 800 to 1100 feet (may also be a lithophysae-bearing subzone at about $850 \mathrm{ft}$.).

4) The base of the lower lithophysal zone of the Topopah Spring Tuff at about 1200 to 1300 feet.

5) The crystal-poor vitric zone near the base of the Topopah Spring Tuff at about 1500 feet.

6) The top of the moderate to densely welded zone of the Prow Pass Tuff at about 2000 feet.

7) The top of the densely welded zone of the Bullfrog Tuff at about 2500 feet.

8) The top of the densely welded zone of the Tram Tuff at about 2700 feet.

A notable feature of line 2 is the apparent lowered block between NRG-5 and NRG-7. This feature agrees with complex variations in formation thickness and block structures mapped along Drill Hole wash. These features are believed caused by bends in the north-west trending strike-slip Drill Hole Wash fault, and north-east trending faults as mapped on Azreal Ridge (Buesch, et al, 1994, and see Fig 13).

We have intentionally avoided interpreting faults on line 2 because line 2 is parallel to the major fault features in Drill Hole Wash. Additionally, Drill Hole Wash is known to be a much more complex environment than line 1 with a three dimensional nature to the structure (Buesch, et al, 1994). This orientation allows for faults to intersect the line at a range of angles from steep to very shallow and the discontinuities in reflectors make identification of widely ranging fault dips very difficult. In a region of such structural complexity, the identification of true fault dip would require 3-D type seismic data, or at least a perpendicular seismic line. The seismic data from line 2 does indicate faulted offset of formations and variation in bed thickness. The variation in bed thickness could result from both faulting and true thickness changes (Buesch, et. al., 1994).

\section{NRG-6 VSP Analysis}

The well NRG-6 was used for VSP studies to complement the Drill Hole Wash seismic line and to aid studies of rock properties for the ESF. The VSP work was similar to that described for WT-2, except that a second offset VSP was acquired instead of a walkaway survey. Fig 13 shows a map of Drill Hole Wash with the VSP offset locations. The NRG-6 VSP studies focused on:

1) Velocity measurement from zero-offset VSP.

2) Generation of reflection seismogram from zero-offset VSP.

3) VSP-CDP mapping from the two offset source locations.

4) Comparison of the two offset source locations for possible fracture-induced seismic anisotropy.

After performing velocity analysis (reported in Daley and Majer, 1993), the results were compared to velocities measured in NRG-6 cores by New England Research, Inc. (Randolph Martin, personal communication, 1993). Figs $18 \mathrm{a}$ and $\mathrm{b}$ show a comparison of VSP and core velocities. The VSP velocities are notably slower than the core velocities. We interpret this 
difference to mean the longer wavelengths of a VSP study ( about $100 \mathrm{ft}$. compared to about 1 inch for core measurements) are sampling more fracturing in the rock and larger scale fracturing than sampled by cores. The fracturing would decrease the velocity. Also, the cores can not physically sample the lithophysae which can be many centimeters in diameters. We believe the scale of physical properties measured by VSP and surface seismic is more appropriate for engineering design of large diameter tunnels.

The reflection seismogram from the zero-offset P-wave survey is shown in Fig 19. We see partial agreement between the VSP reflection seismogram and line 2 near NRG-6, and while there are some reflectors which do not tie, we gain initial confidence for the interpretation of line 2.

The VSP-CDP sections for offsets 2 and 3 are shown in Figs 20a and $b(P$ and $S)$ and 21a and $b(P$ and $S)$. We did not use the same scale as line 2 for these sections because the offsets and well depth are too small. However, we did make a reflection seismogram from each of the offset VSP-CDP data sets for comparison with line 2 (Figs 22a and b, and 23a and b). While there is variation in reflectivity between offsets (which reflects the heterogeneous nature of Drill Hole Wash), there is general agreement with reflectors identified on line 2 such as the reflector at about $1100 \mathrm{ft}$. at NRG-6.

The comparison of offsets 2 and 3 for seismic anisotropy was reported in Daley and Majer (1993) and it was most notable for the variation in shear-wave polarization. Offset $3 \mathrm{~S}$-wave data was polarized along the line connecting NRG-6 and offset 3 . As can be seen in Fig 13, this line is parallel to the local trace of Drill-Hole Wash fault and it seems most likely that a fault trace is causing the shear-wave polarization anomaly.

\section{Line 3 - Yucca Crest}

As stated earlier, line $3 \mathrm{had}$ the poorest data quality of the three reflection lines. Poor field conditions (including winds and hard rock outcrops) and limited time available for acquisition combined to reduce the data quality. We did follow the same processing sequence used for lines 1 and 2 to produce a CDP stack for line 3. This stack is shown in Fig 24 in a time section. No attempt was made to convert line 3 to a depth section because we did not feel it could be adequately interpreted and there were no borehole surveys along line 3 to guide time-depth conversion. We do feel there is enough reflected energy in lines 1 and 2 to encourage another attempt at data acquisition on Yucca Crest. However Line 3 is too noisy and incoherent to attempt interpretation.

\section{Summary and Conclusions}

We believe these studies demonstrate the positive potential of seismic reflection imaging in and around Yucca Mountain. The combined use of VSP and surface reflection profiling, in collaboration with geologists, allows an interpretation of lithologic and structural setting to be defined near a well and then extrapolated away from the well. We would like to emphasize the importance of interaction between geophysicists and geologists during survey design and data interpretation. The Ghost Dance fault studies of line 1 show that the fault system has multiple vertical features to the east of well WT-2 which offset units down to the west. The seismic reflections observed can be correlated with lithology, however the seismic reflections are discontinuous. The maximum reflector lateral continuity observed is approximately 1000 feet. The frequent seismic reflector discontinuities hampered accurate interpretation of fault dip. 
The Drill Hole Wash study provided useful information about the complicated subsurface structure. Our VSP results indicate aligned fracturing/faulting to the northeast near NRG-6 and a complicated set of faults and/or seismic discontinuities along line 2 . We believe the seismic section images the variation in subsurface formation thickness previously interpreted from surface and drillhole geologic studies (Buesch, et. al., 1994).

We believe further seismic data acquisition, utilizing the results of the study, could give better results. We recommend a smaller spread length with smaller sensor spacing; this shortening would allow penetration between the seismic discontinuities and also give better spatial sampling of noise sources (such as ground roll and air waves). Additionally, more source energy (either larger sources or more stacks) should be used. The VSP surveys, which utilized large vibroseis trucks, had better signal-to-noise characteristics than the surface seismic. Of course, the large vibroseis trucks have lower frequency response and less resolution as seen in the VSP data. We feel this study is a successful first step in learning optimal application of seismic imaging techniques to the characterization of Yucca Mountain. The relative success of this work was aided by high frequency content (64-150 Hz), high dynamic range ( 24 bit recording), and our previous experience with data acquisition in tuffs at Rainier Mesa (using source arrays and pushing the line).

The major conclusions regarding the character of the geologic structure from using both the VSP and surface reflection data are:

1) The geologic structure is 3-D in nature and laterally heterogeneous at scales on the order of 500 to 1000 feet.

2) A variety of factors point to a highly fractured, fault controlled environment including the following:

a) The 3-component results from the P-and S-wave VSP work reported in Daley and Majer, 1993, shows anisotropy to the depths of the VSP that is indicative of fracturing/faulting directions consistent with the mapped surface faulting.

b) The slower velocities from the VSP results compared to the core and well-log velocities indicate that fracturing is the cause, rather than matrix variability.

c) The surface reflection and VSP results are consistent with the gravity and magnetic results (Oliver, et al, 1994). The magnetic results are consistent with the seismic in that both allow for faulting in the same general locations and with the same approximate spacing between faults.

d) Offsets in reflectors which correlate at different depths indicate that the discontinuities in reflectors are due to vertical cross-cutting features rather than lateral changes in matrix properties.

e) In regions where we have the most data (WT-2 to UZ-16) the VSP, surface seismic profile, well-logs, gravity, and magnetics, all indicate through going vertical features.

f) In both the VSP and surface reflection data we see that seismic energy is best propagated vertically through the earth and very poorly propagated laterally through the earth at distances over 500 to 1000 feet. This observation is strongly indicative of highly attenuating through-going vertical features.

3) At this point the data allow for both a linear and listric fault model.

This was the first attempt at high resolution seismic imaging using both VSP and surface reflection data in a combined fashion (McGovern, et. al., 1983). Modern wide band, high dynamic range (24 bit) data recording systems coupled with a high frequency impact source provided successful results. Taken alone the VSP and/or surface reflection results would be suspect, or at least difficult to process and interpret. Great care was taken not to "over-process" or process to a point where results were "forced" on the data. When the VSP and surface reflection results are 
combined with the available surface geologic mapping, well-logs, core analysis, magnetics and gravity, an image emerges that indicates a layered structure interrupted with through-going vertical features. Some of the seismic features are obviously due to variability in rock matrix structure, but other evidence points to faulting as the cause of these vertical features.

\section{Acknowledgments}

The authors would like to thank Rick Spengler and David Buesch of the U.S.G.S. for their input and reviews. The VSP data acquisition was greatly assisted by Don Lippert and Ramsey Haught of LBL's Geophysical Measurement Facility. Data Processing was performed at LBL's Center for Computatational Seismology. 


\section{References}

Balch, A.H., and Lee, M.W., editors, 1984, Vertical Seismic Profiling: Technique, Applications, and Case Histories, IHDRC, Boston MA.

Buesch, D.C., Dickerson, R.P., Drake, R.M., and Spengler, R.W., 1994, Integrated geology and preliminary cross section along the north ramp of the exploratory studies facility, Yucca Mountain, in Proceedings of the Fifth Annual International Conference on High Level Radioactive Waste Management, Las Vegas, Nevada, volume 2.

Daley, T.M. and Majer, E.L., 1993, Analysis Paper: Data Reduction NRG-6/WT-2 VSP, Interim Report for Activity: 8.3.1.4.2.2.5, Yucca Mountain Project.

Gelsin, J.K., Moyer, T.C., and Beusch, D.C., in press, Summary of lithologic logging of new and existing boreholes at Yucca Mountain, Nevada, August 1993 to February 1994, U.S. Geological Survey Open File Report 94-342.

Gelsin, J.K., Moyer, T.C., 1994, written communication, Graphical lithologic log of borehole NRG-7/7A, DTN: GS940408314211.020.

Harding, S.T., 1988, Preliminary results of high-resolution seismic-reflection surveys conducted across the Beatty and Crater Flat fault scarps, Nevada, in Geologic and Hydrologic Investigations of a Potential Nuclear Waste Disposal Site at Yucca Mountain, Southern Nevada, M.D. Carr and J.C. Yount (editors), U.S. Geological Survey Bulletin 1790, p. 121127.

USGS Rock Characteristics Section, 1994, written communication through L.R. Hayes to E.L. Majer, Methodology and Source Data used to construct the demonstration lithostratigraphic model: Second Progress Report, and L.R. Hayes to S. Jones, Early submittal of 3-D site scale lithostratigraphic model, version YMP.R1.0

Please be advised that there may be uncorrected errors within this preliminary model as no quality checking or technical review has been performed on this version, YMP.R1.0

Majer, E.L. and Karageorgi, E., 1993, Analysis Paper: Ghost Dance Surface Reflection Profiles, Milestone Report for Activity 8.3.1.4.2.2.5. Yucca Mountain Project.

Majer, E.L., Johnson, L.R., Karageorgi, E.K., Peterson, J.E., 1994, High-resolution seismic imaging of Rainier Mesa using surface reflection and surface-to-tunnel tomography, in Proceedings of the Symposium on the Non-Proliferation Experiment Results and Implications, M.D. Denny et. al., editors, Lawrence Livermore National Laboratory, Livermore, CA, CONF-9404100.

Martin III, R.J., 1993, personnel communication, Summary data sheet: NRG-6 Borehole.

McGovern, T.F., Pankratz, L.W., and Ackerman, H.D., 1983, An evaluation of seismic reflection studies in the Yucca Mountain area, Nevada Test Site, U.S. Geological Survey Open-file Report 83-912.

Nelson, P.H., Muller, D.C., Schimschal, U., Kibler, J.E., 1991, Geophysical logs and core measurements from fourty boreholes at Yucca Mountain, Nevada, U.S. Geological Survey, Geophysical Investigations, Map GP-1001, 64p.

Oliver, H.W., Majer, E.L., and Spengler, R.W., 1994, Geophysical investigations of the Ghost Dance Fault, Yucca Mountain, Nevada, abstract in Geological Society of America Cordilleran section meeting, San Bernardino, Ca., March 1994, p.78.

Scott, R.B. and Bonk, J., 1984, Preliminary geologic map of Yucca Mountain, Nye County, Nevada with geologic sections, U.S. Geological Survey Open File Report 84-494. 
Spengler, R.W. and Fox Jr., K.F., 1989, Stratigraphic and structural framework of Yucca Mountain, Nevada, Radioactive Waste Management and the Nuclear Fuel Cycle, vol 13(1-4), pp 21-36.

Spengler, R.W., 1994, personnel communication to authors. 
TABLE 1

Coordinates of some reference points along surface seismic reflection lines 1 and 2 .

Original Coordinates on NAD 83 Geographic Coordinates Translated Coordinates below on NAD 27 State Plane Zone 2702, U.S. FOOT *Elevations are in meters, with respect to mean sea level.

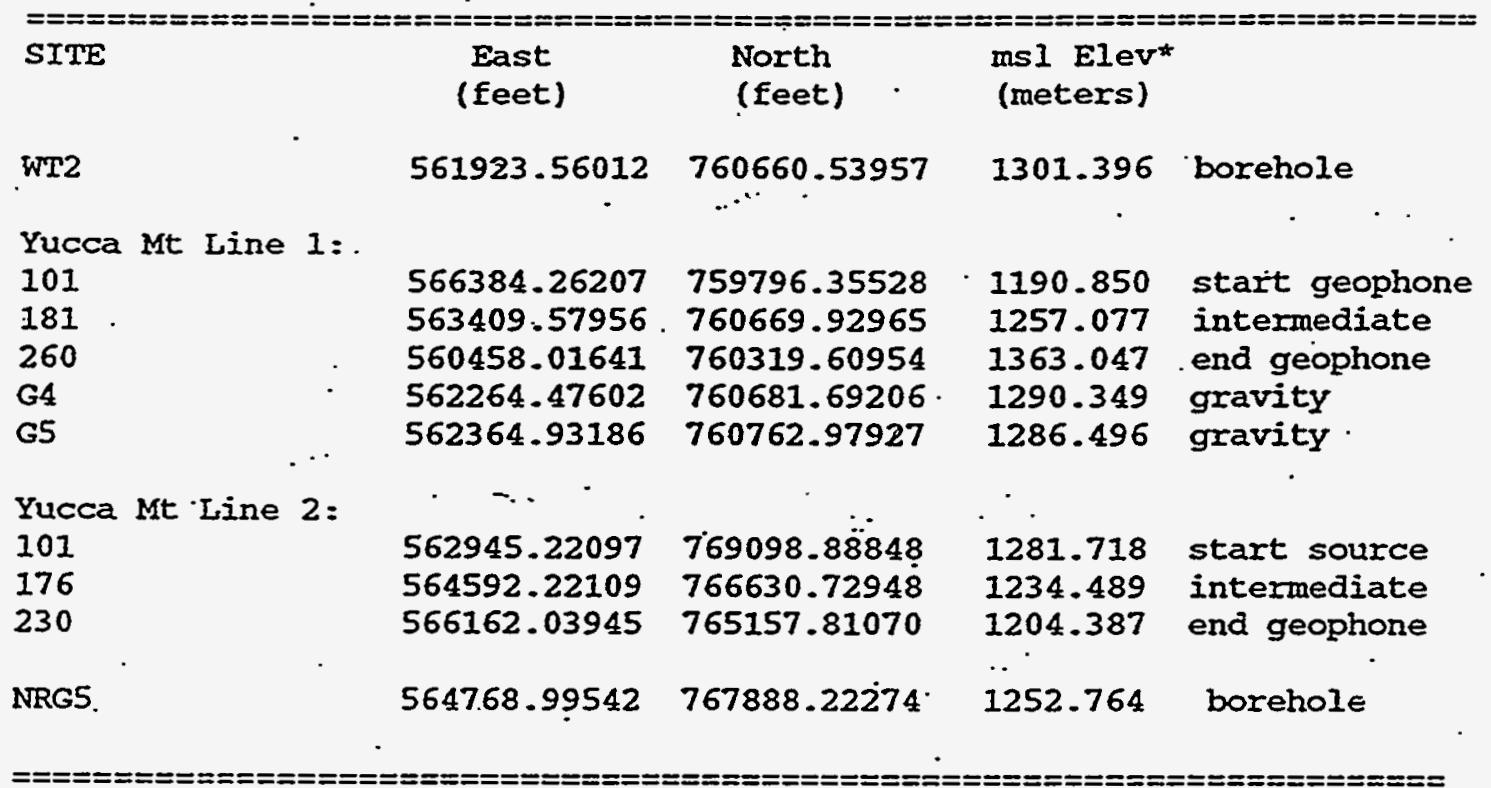




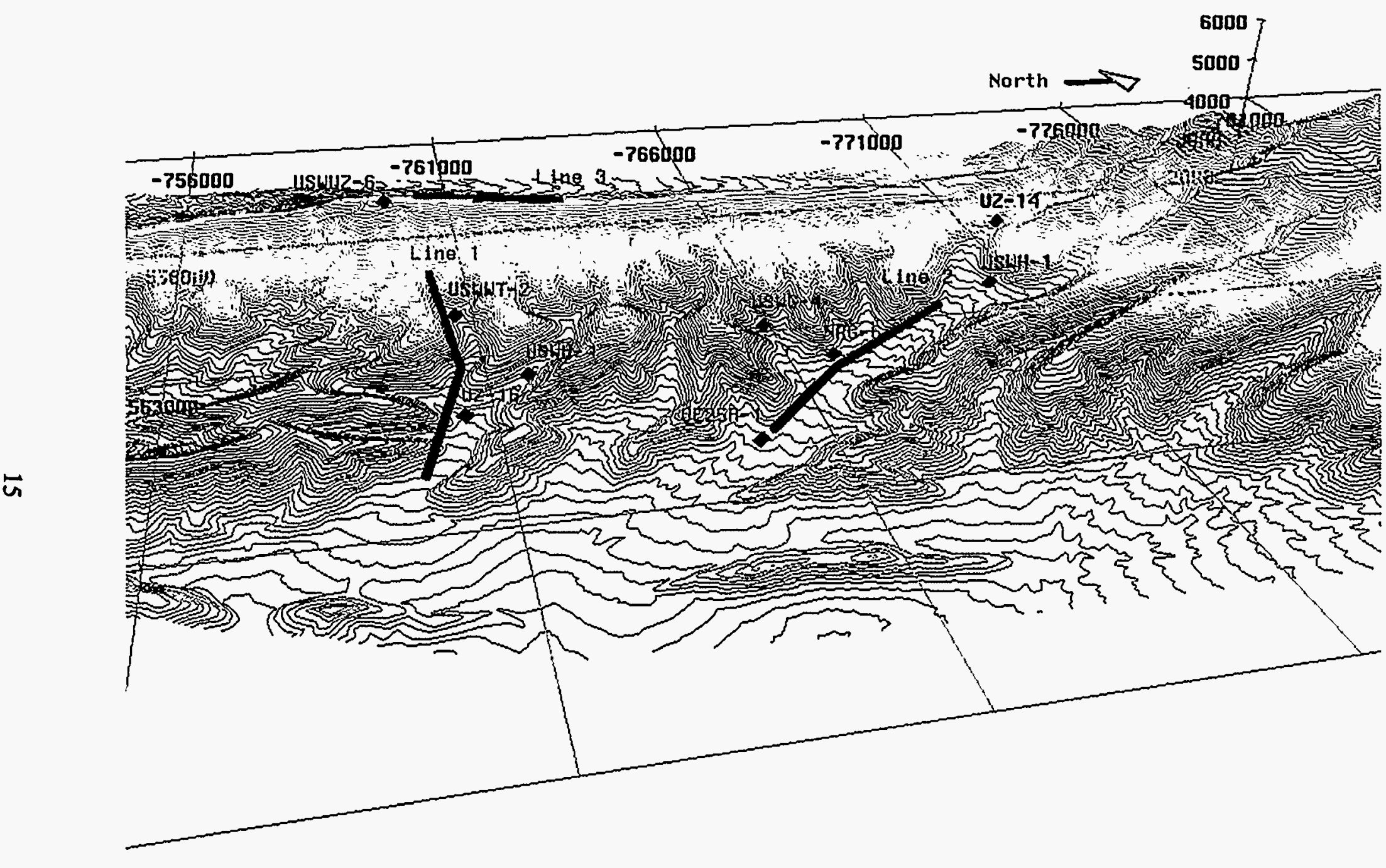

Figure la Topographic map of Yucca Mountain showing location of seismic lines 1,2 and 3 (in red) in relation to topography and some well locations (in blue). The grid is 5000 foot spacing and the coordinates are Nevada state plane. Note that this is a perspective view and is not 1:1 scale. 

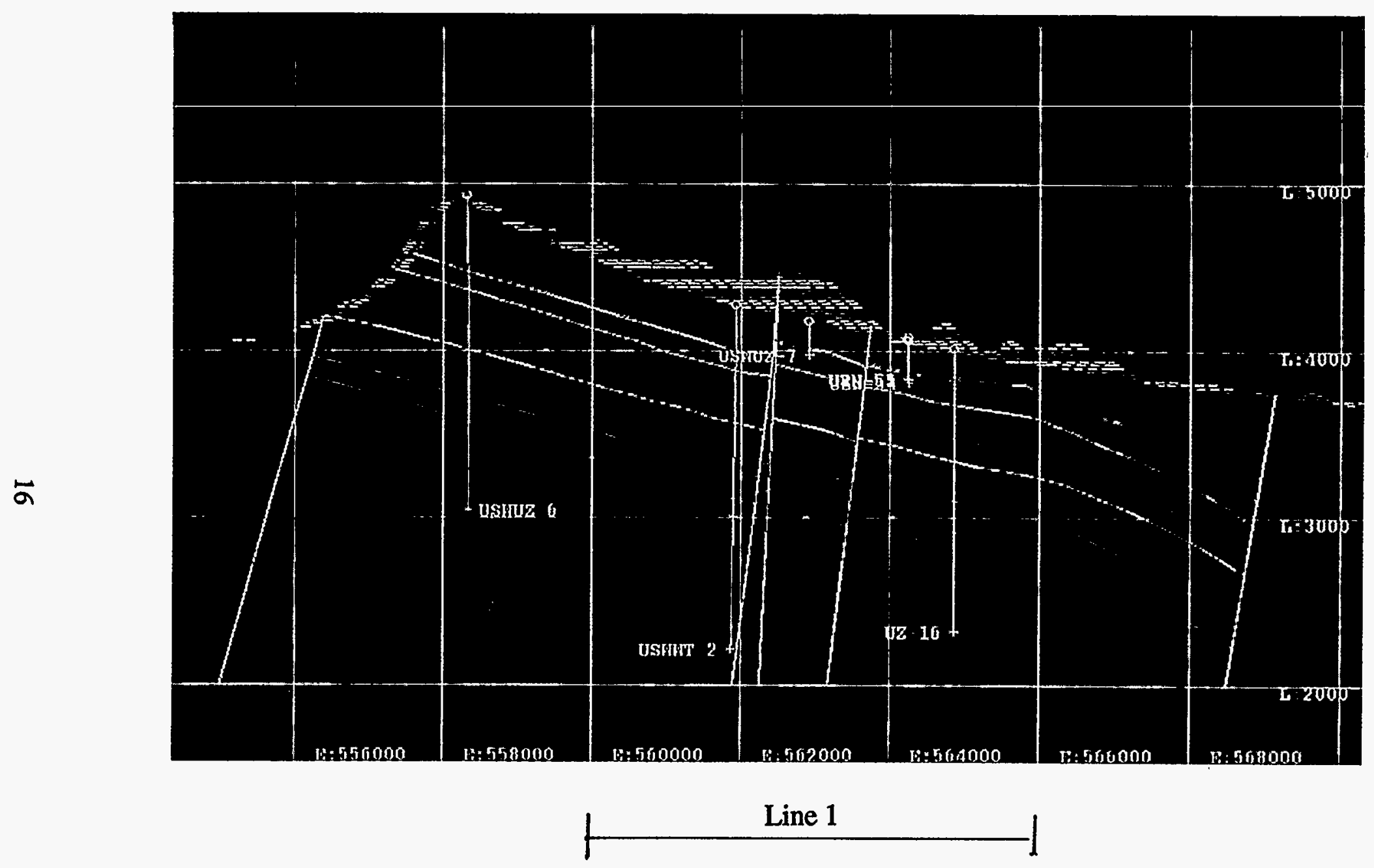

Figure $1 \mathrm{~b}$ Cross section along seismic line 1 showing faults, nearby wells and geologic horizons between the top of the crystal-poor vitric zone of the Tiva Canyon Tuff (Tpcpv, top red line) and the base of the crystal-poor vitric zone at the base of the Topopah Spring Tuff (Tptpv, bottom red line). From USGS Rock Characteristics Section, 1994. Please note that there may be uncorrected errors within this preliminary model since quality checking and technical review have not been performed on this preliminary version. 


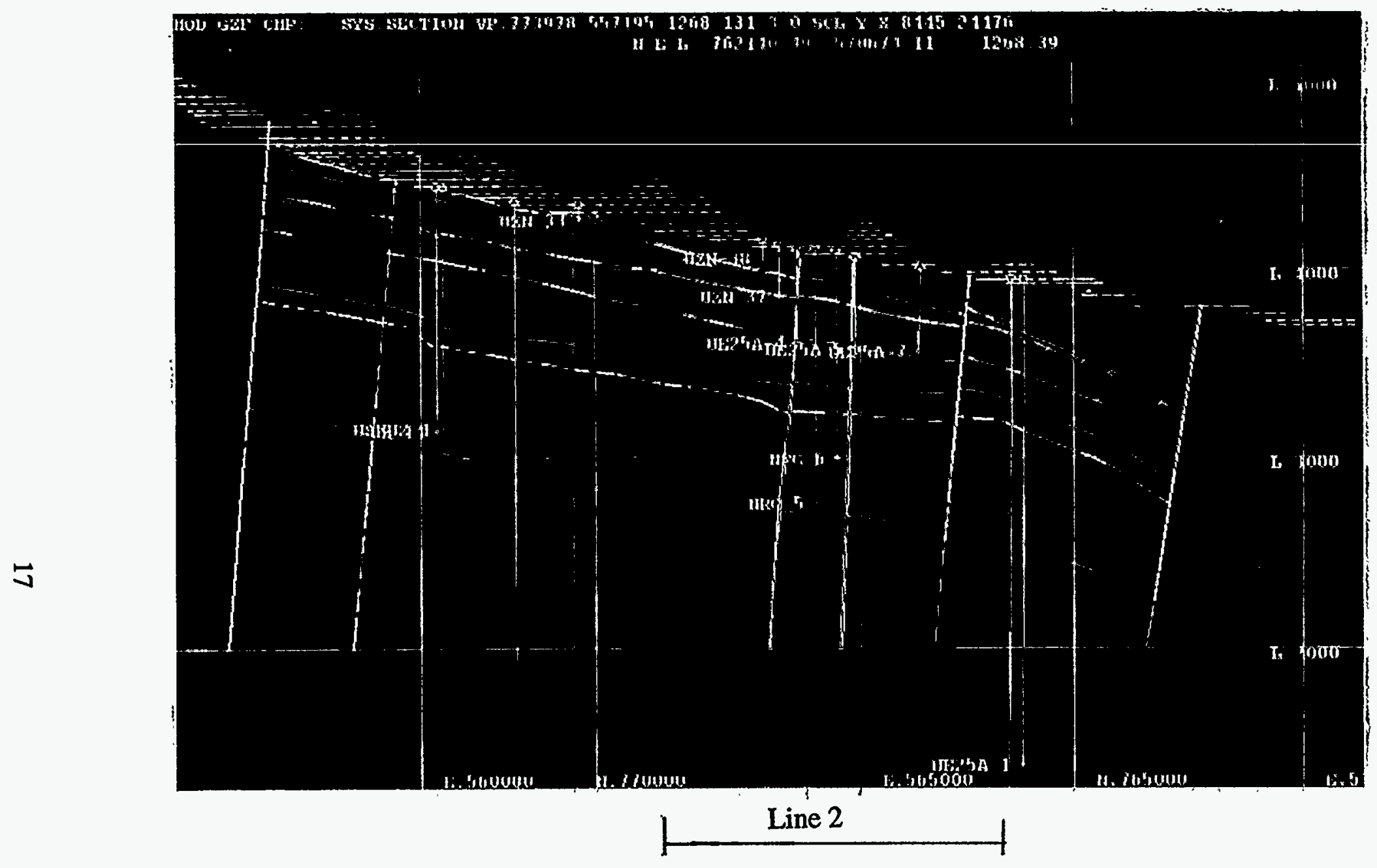

Figure 1c Cross section along seismic line 2 showing faults, nearby wells and some geologic horizons between the top of the crystal-poor vitric zone of the Tiva Canyon Tuff (Tpcpv, top red line) and the base of the crystal-poor vitric zone at the base of the Topopah Spring Tuff (Tptpv, bottom red line). From USGS Rock Characteristics Section, 1994. Please note that there may be uncorrected errors within this preliminary model since quality checking and technical review have not been performed on this preliminary version. 


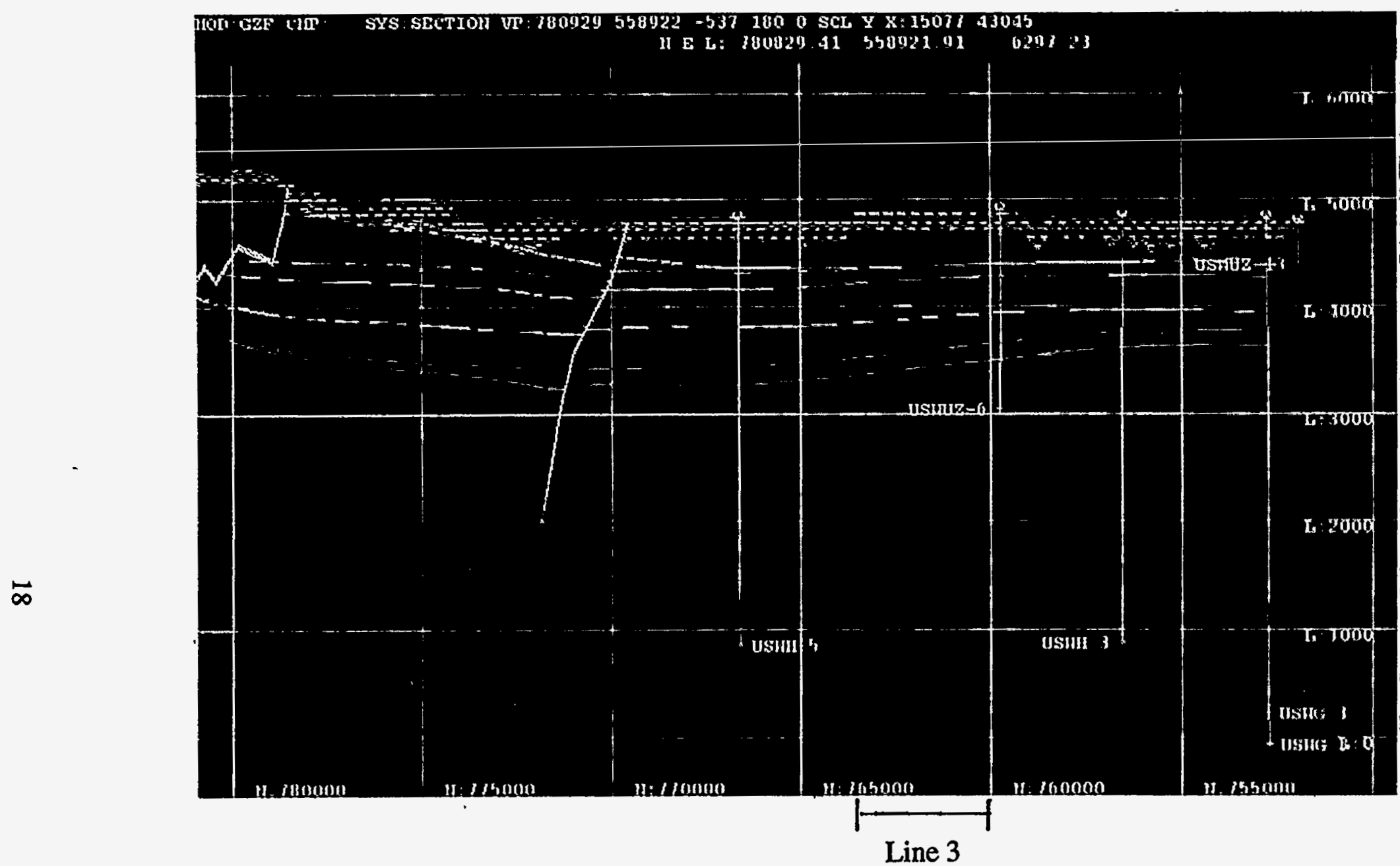

Figure 1d Cross section along seismic line 3 showing faults, nearby wells and some geologic horizons between the top of the crystal-poor vitric zone of the Tiva Canyon Tuff (Tpcpv, top red line) and the base of the crystal-poor vitric zone at the base of the Topopah Spring Tuff (Tptpv, bottom red line). From USGS Rock Characteristics Section, 1994. Please note that there may be uncorrected errors within this preliminary model since quality checking and technical review have not been performed on this preliminary version. 


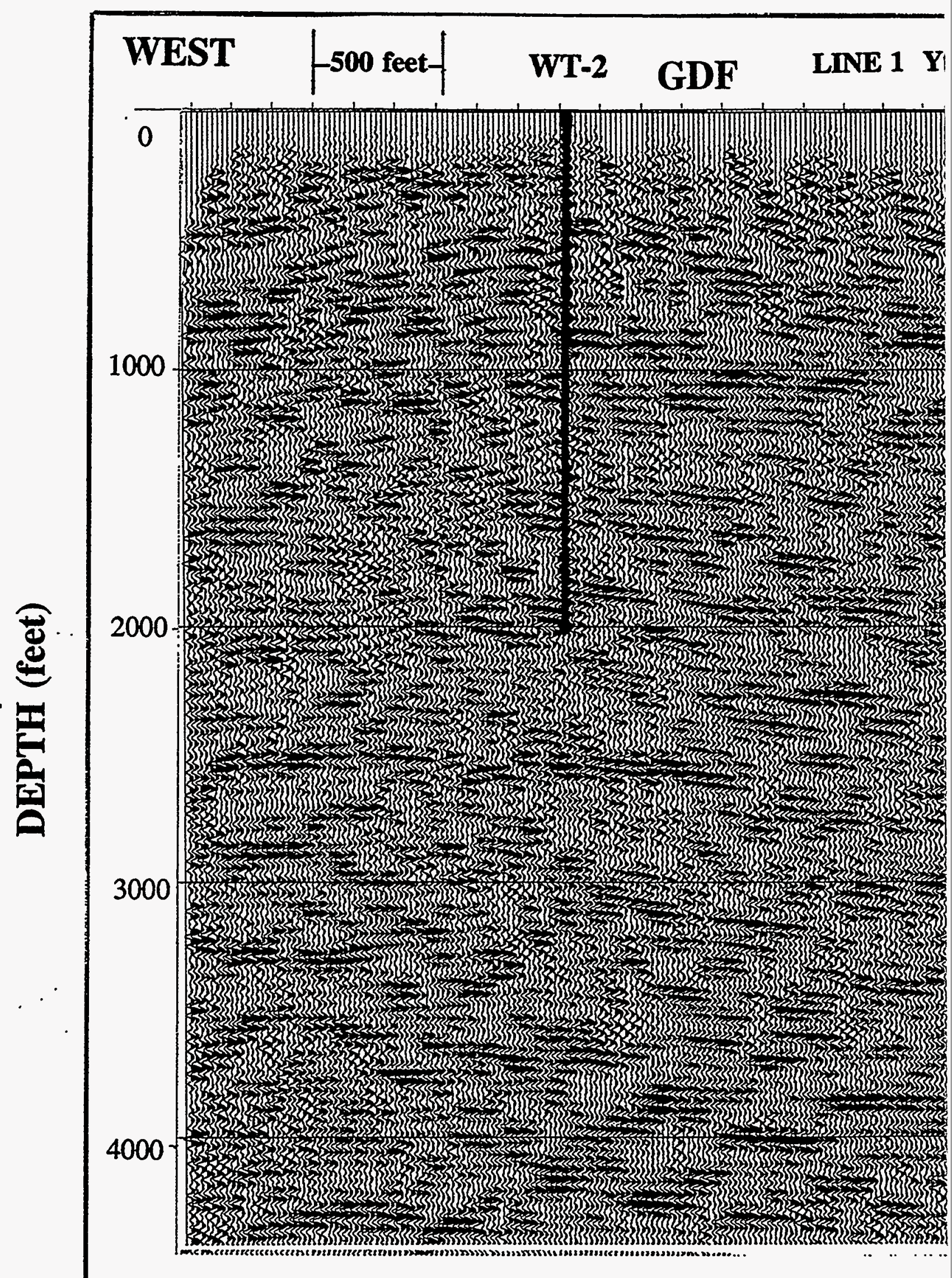

Figure 2 Seismic Line 1. Depth s 


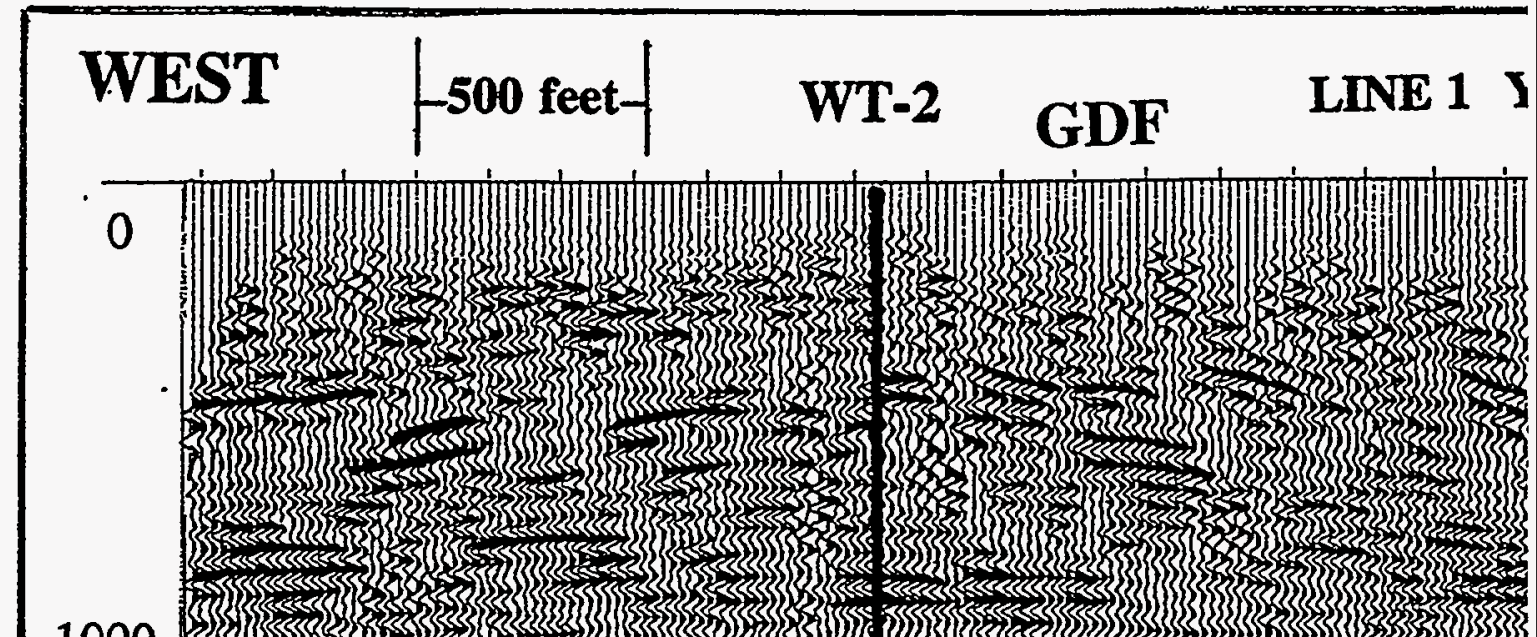

1000 3 3 323

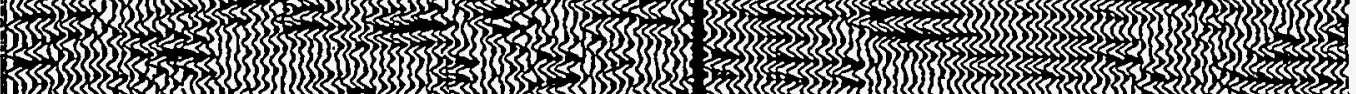

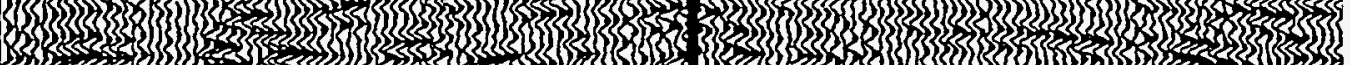
3

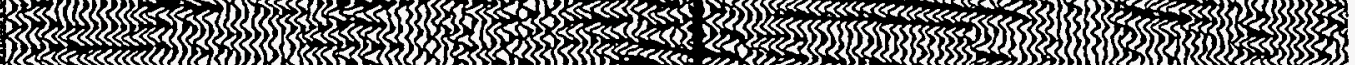
-

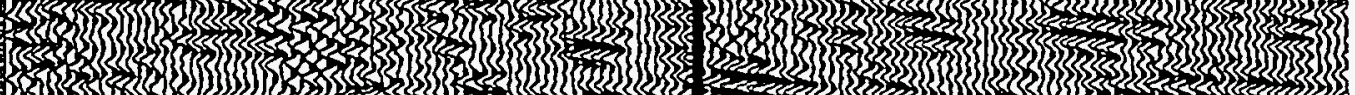

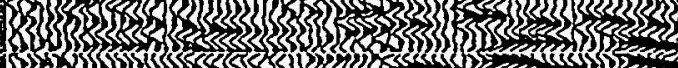

2000

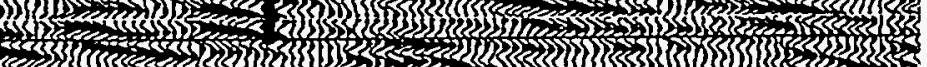
(3) 336-3

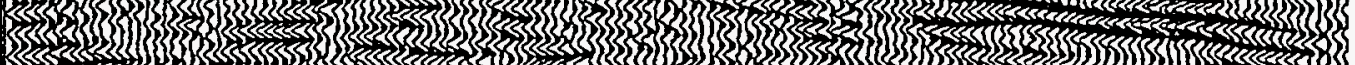

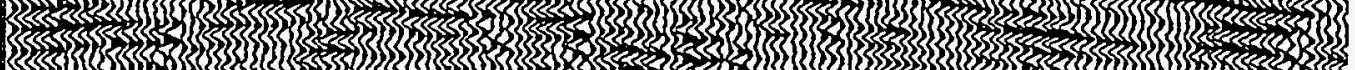
3.3. J

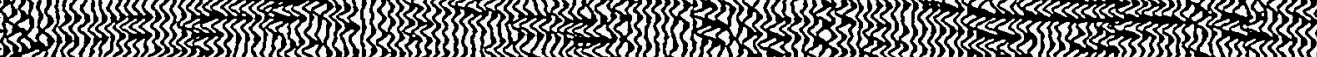

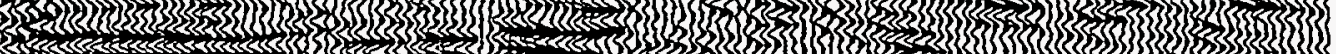
3000 m (3) 3 (3)

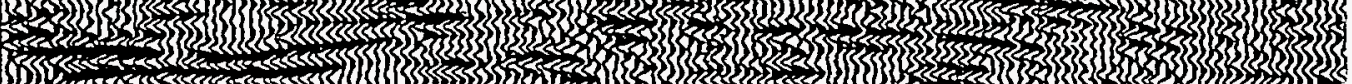

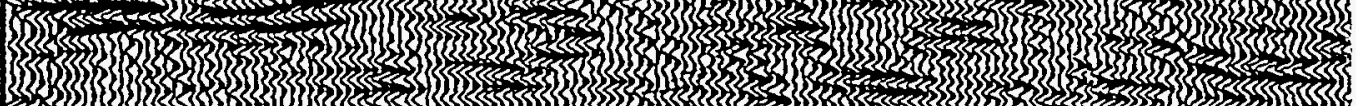

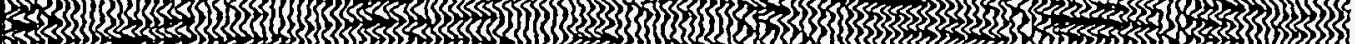
33

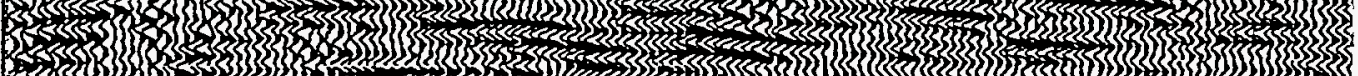

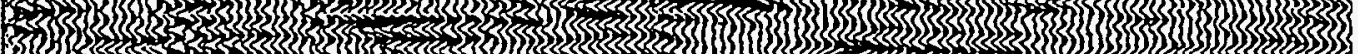

3. 4000 3

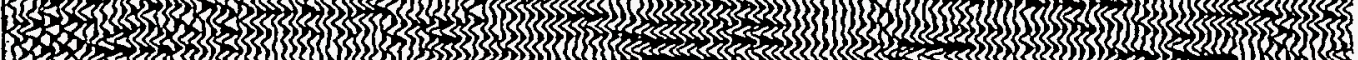

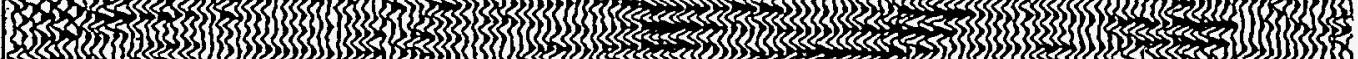
3030303

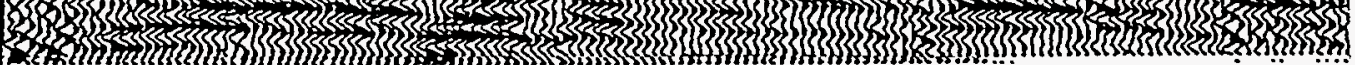

Figure 3 Seismic Line 1. Depth section at 1:6 


\section{DEPTH (feet)}

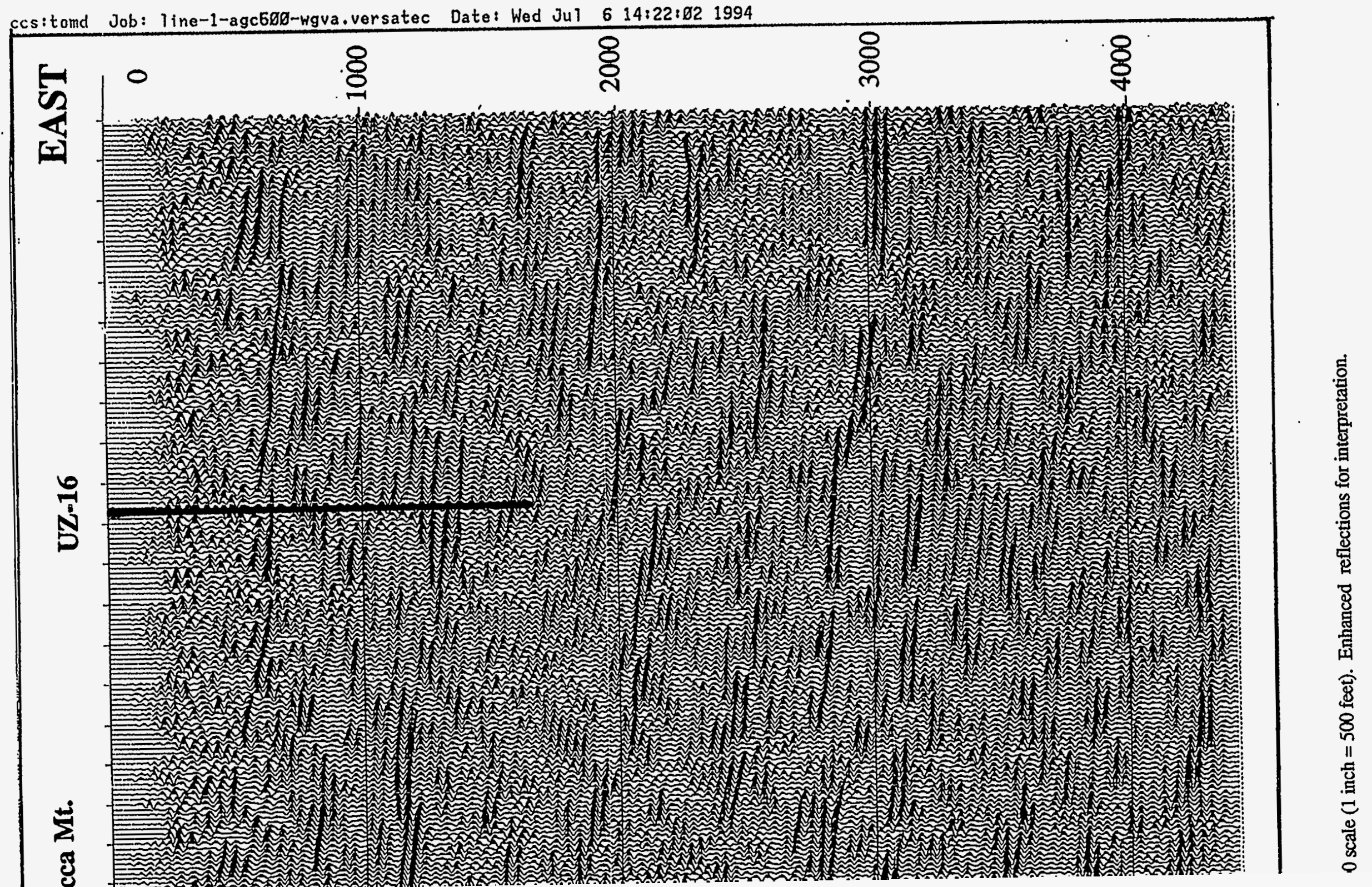




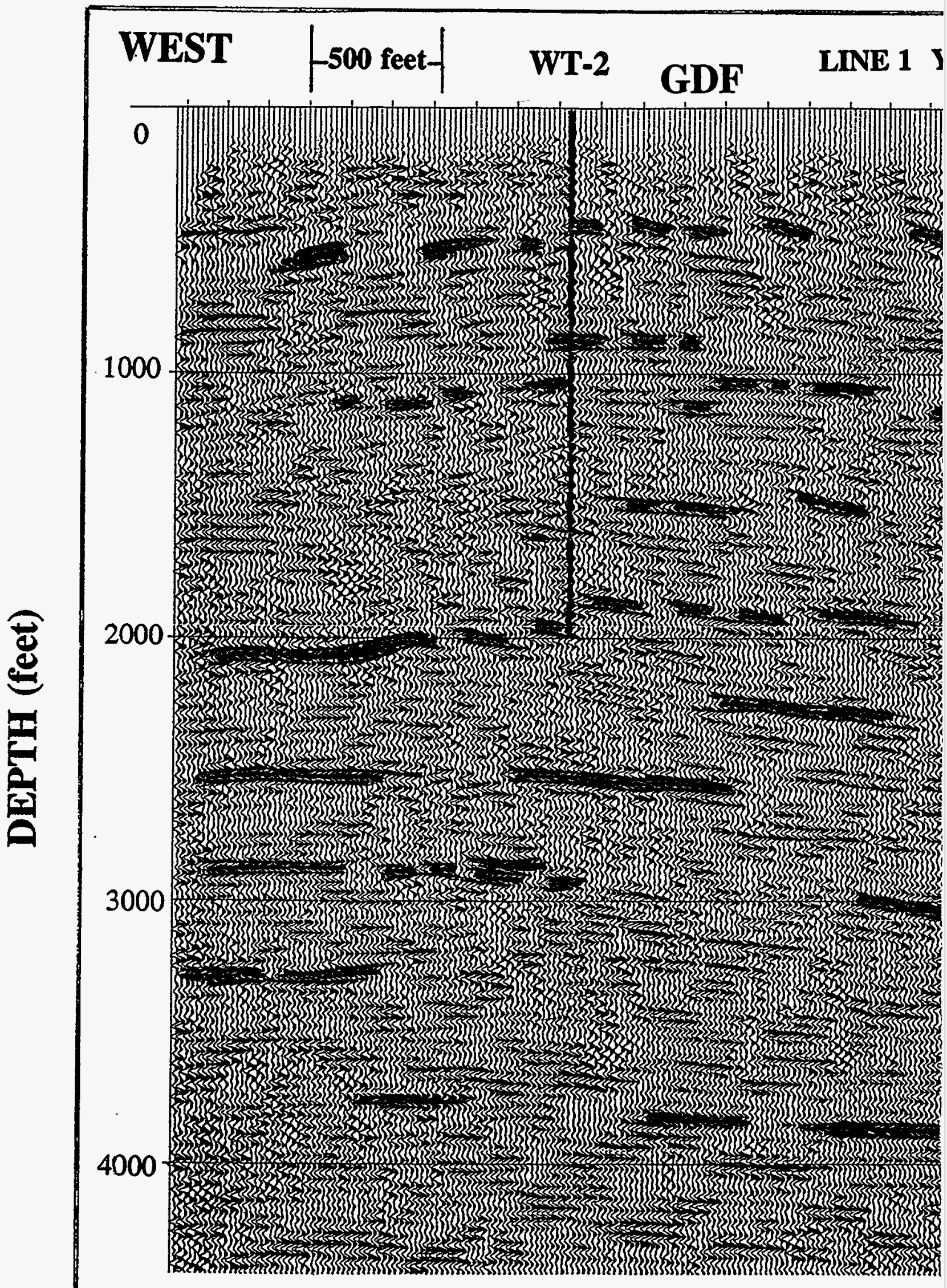

Figure 4 Seismic Line 1. Depth section al 
cea Mt.

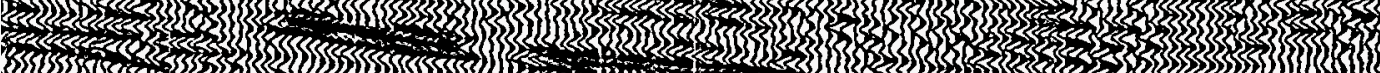
3313 InJ

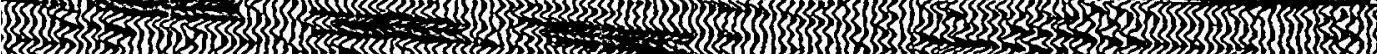
30303030303 FIJ 203

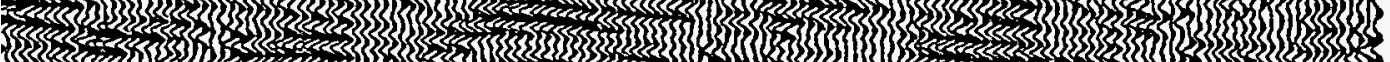

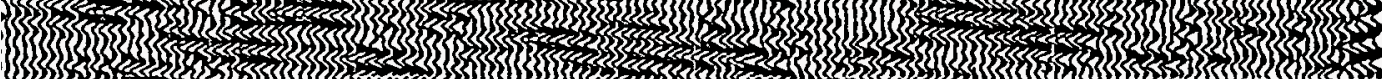

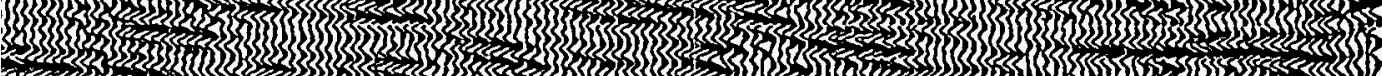

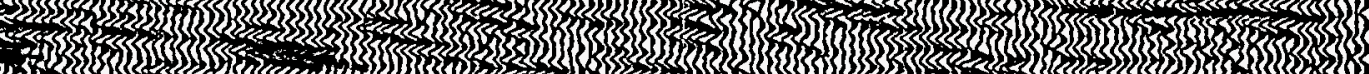

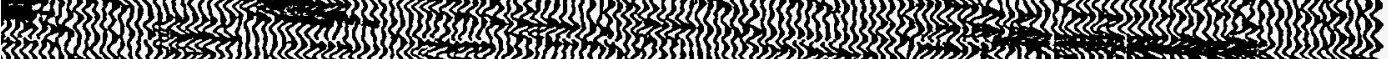

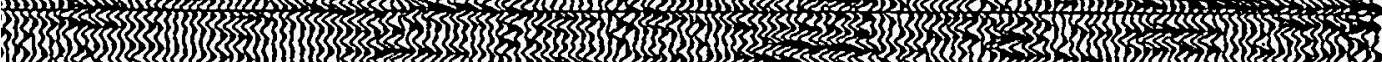




\section{DEPTH (feet)}

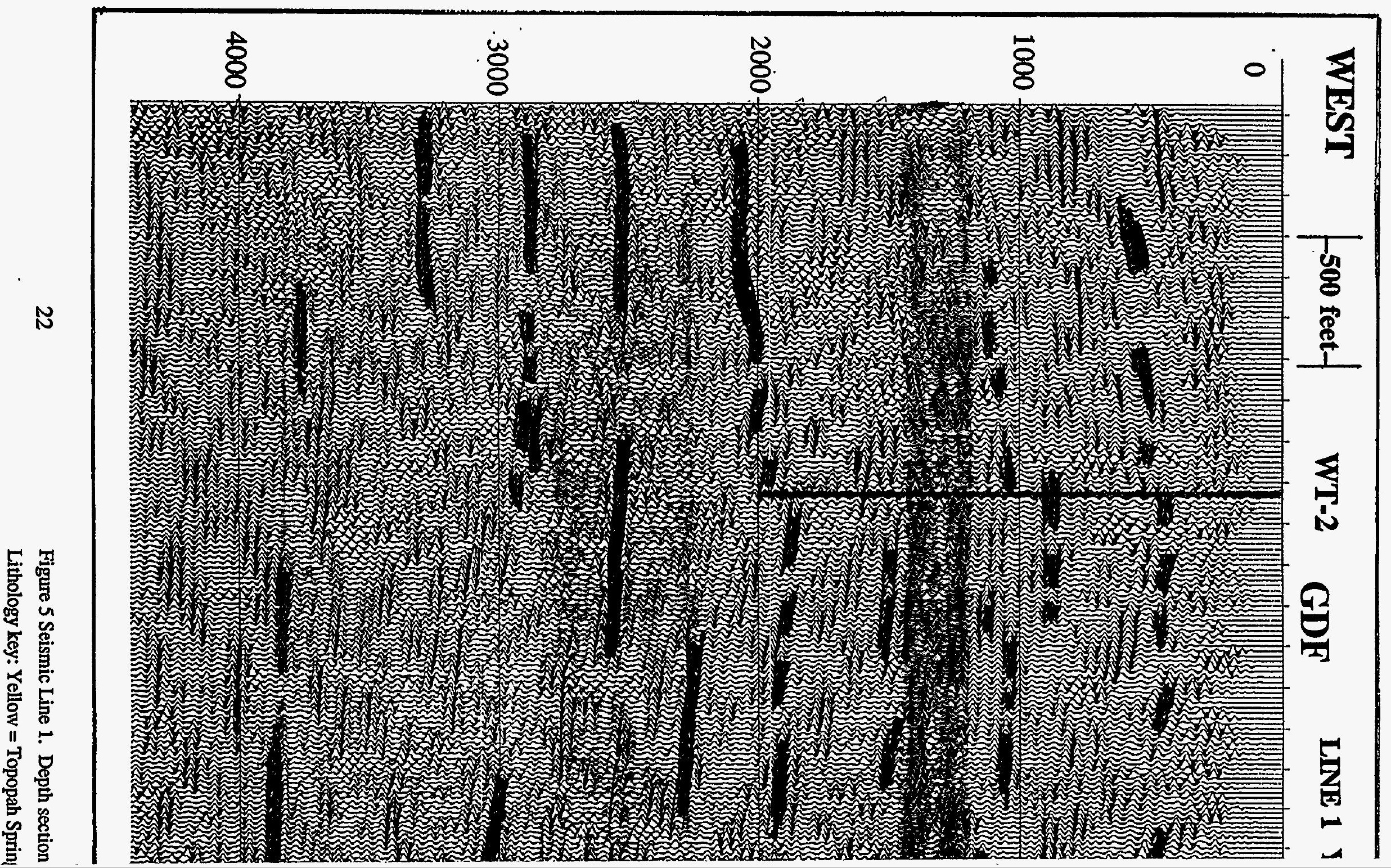


Icca Mt.

UZ-16

EAST

In

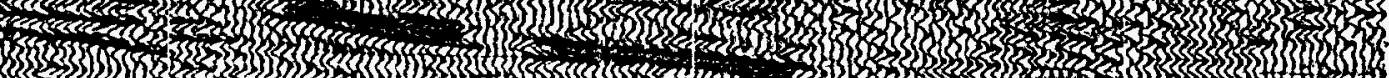

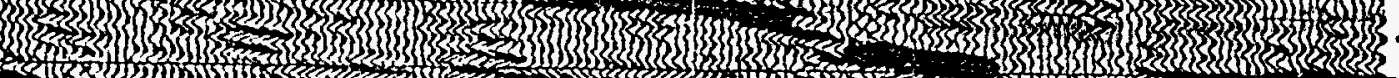

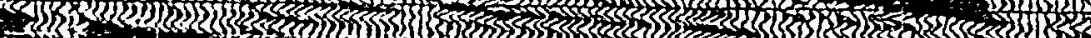

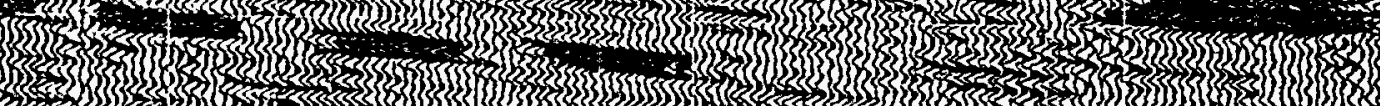

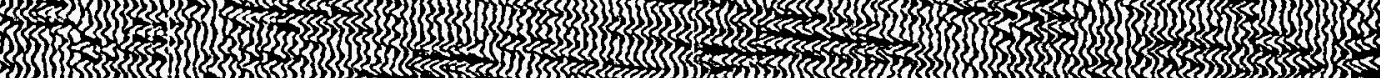
3) 3 J

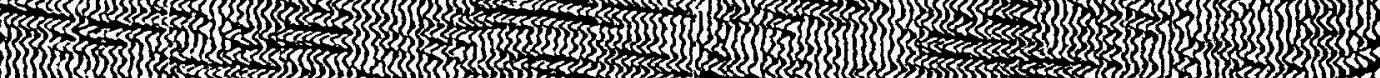

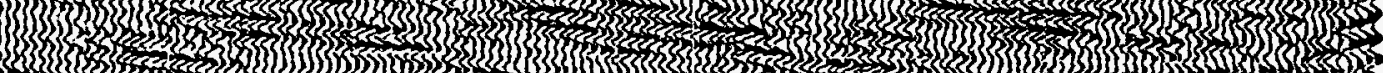

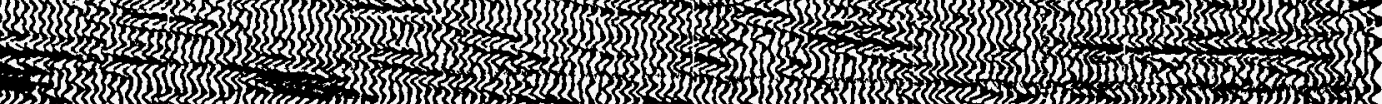

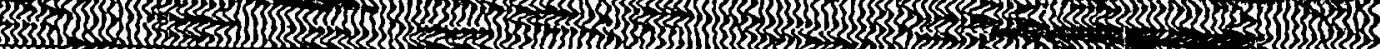
(1) 3

1:6000 scale ( 1 inch $=500$ feet). Notable reflections have been highlighted in red. Lithology has been filled in from nearby well data. ink = Calico Hills Formation, Green = Prow Pass Tuff, Orange = Bullfrog Tuff, Blue = Tram Tuff. 


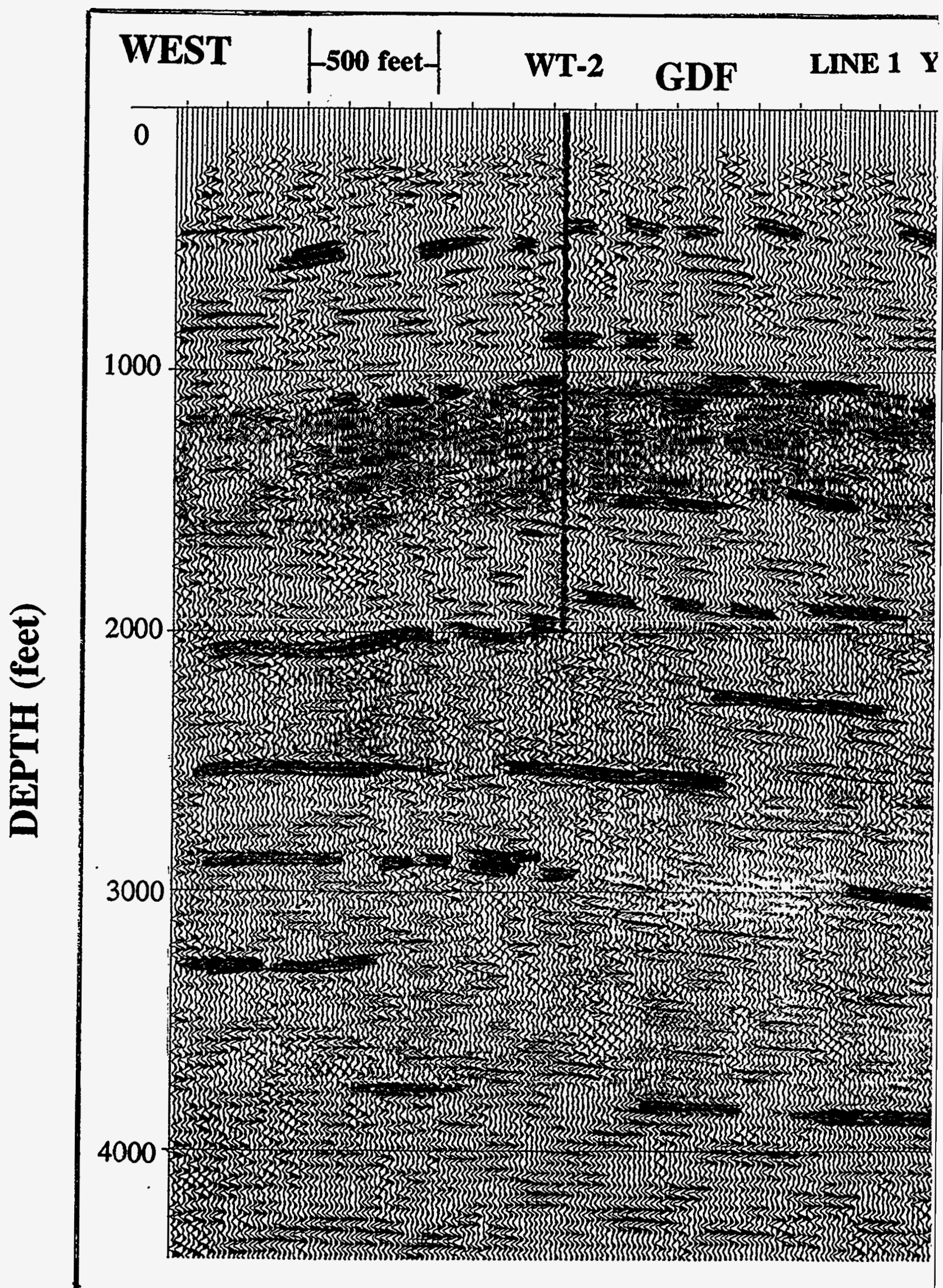

Figure 6 Seismic Line 1. Depth section at : Lithology key: Yellow = Topopah Spring, $P$; 

3 303 3. 3.

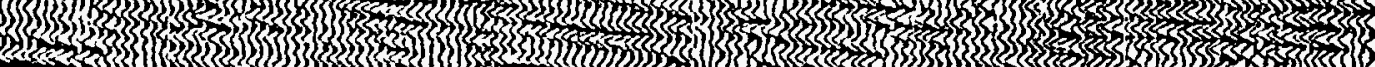

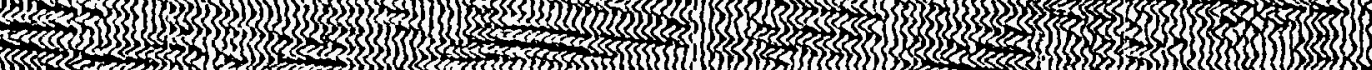
3 OIJ 3. Sor

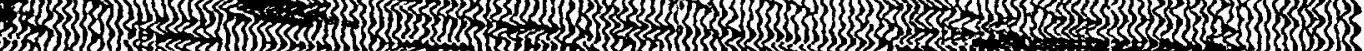

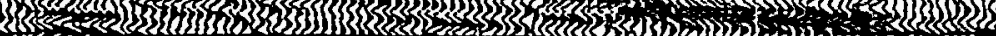

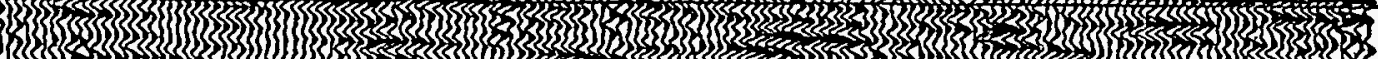

000 scale $(1$ inch $=500$ feet). Notable reflections have been highlighted in red. Lithology has been adjusted to seismic structure. $=$ Calico Hills Formation, Green $=$ Prow Pass Tuff, Orange $=$ Bullfrog Tuff, Blue $=$ Tram Tuff. 


\section{DEPTH (feet)}

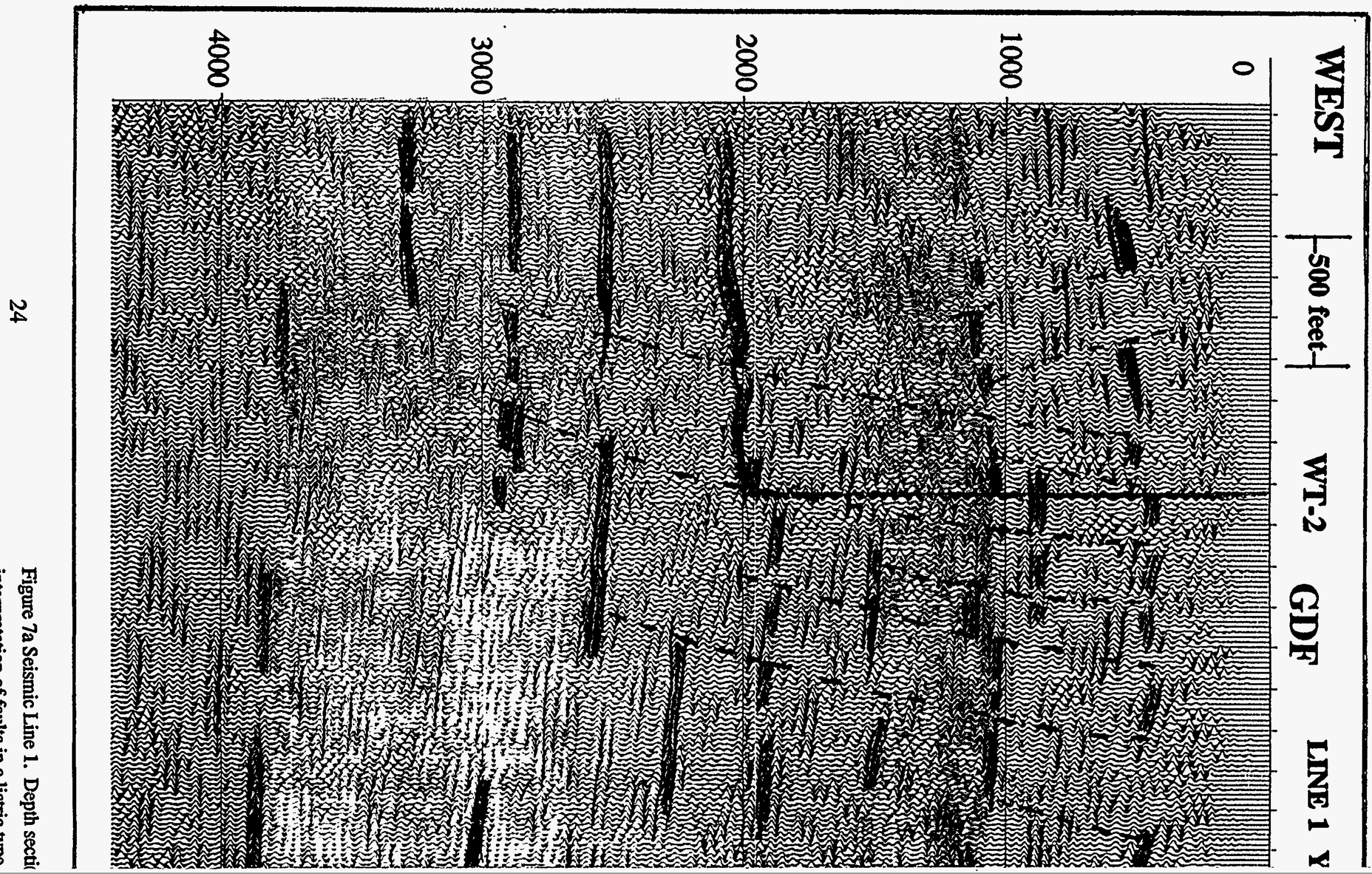




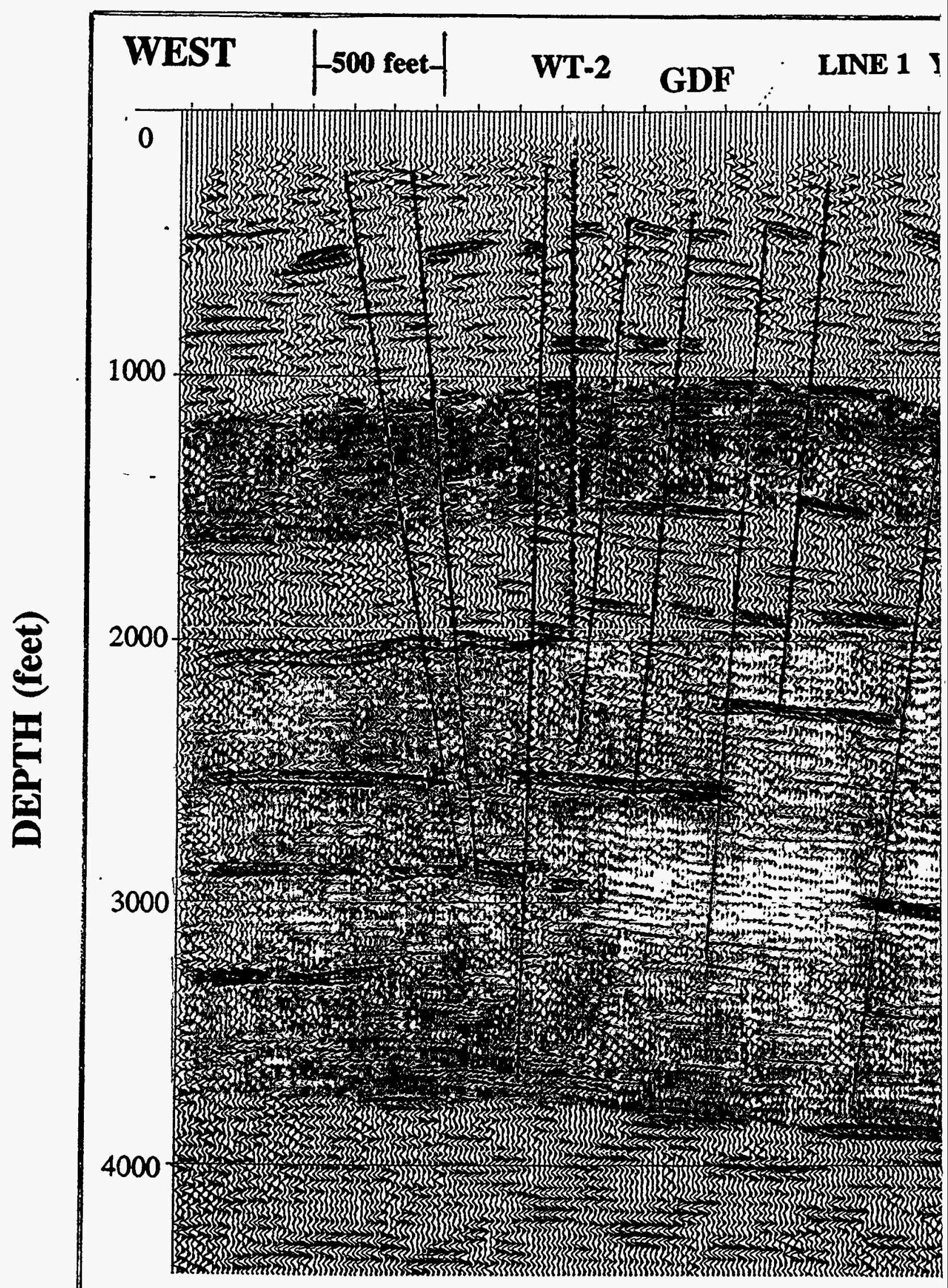

Figure $7 \mathrm{~b}$ Seismic Line 1. Depth sect interpretation of faults in a planer typ Bullfrog Tuff, Blue = Tram Tuff. 

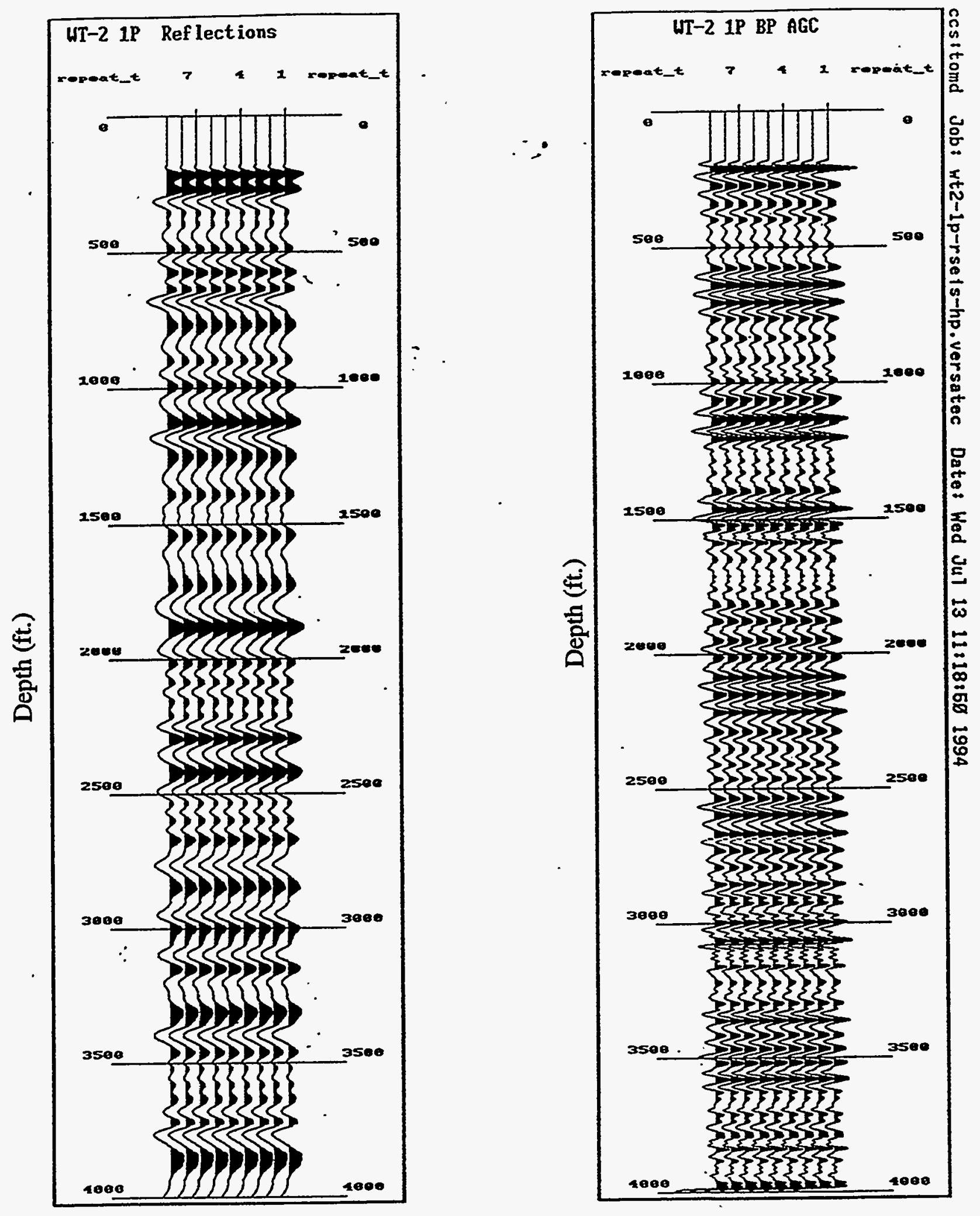

Figure 8a (left) Reflection seismogram from WT-2 VSP zero offset (on well pad) P-wave source. Offset labeled in feet from well.

Figure $8 \mathrm{~b}$ (right) Reflection seismogram from WT-2 VSP zero offset (on well pad) P-wave source. High pass filtered for better comparison to line 1 . 


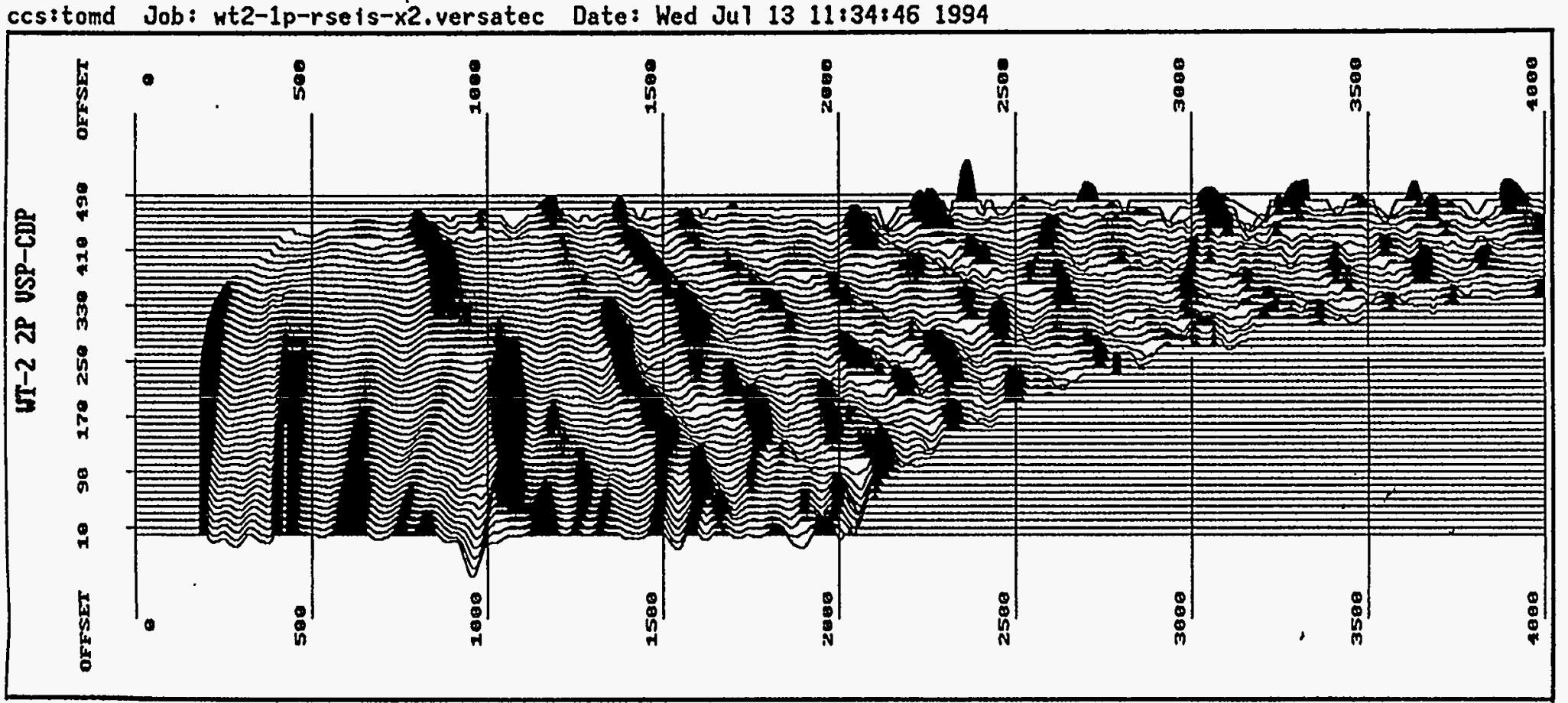

('II) чpdəd

ccsitamd Job: 773973218 Date: Mon Jul 11 17:36:28 1994

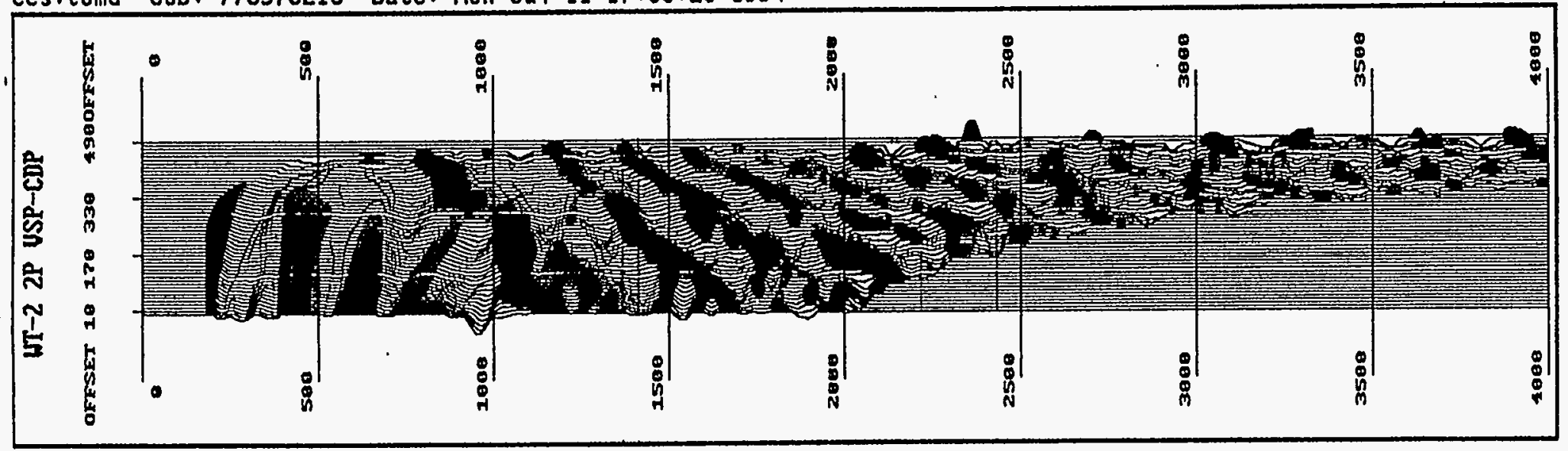

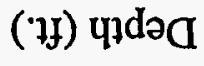




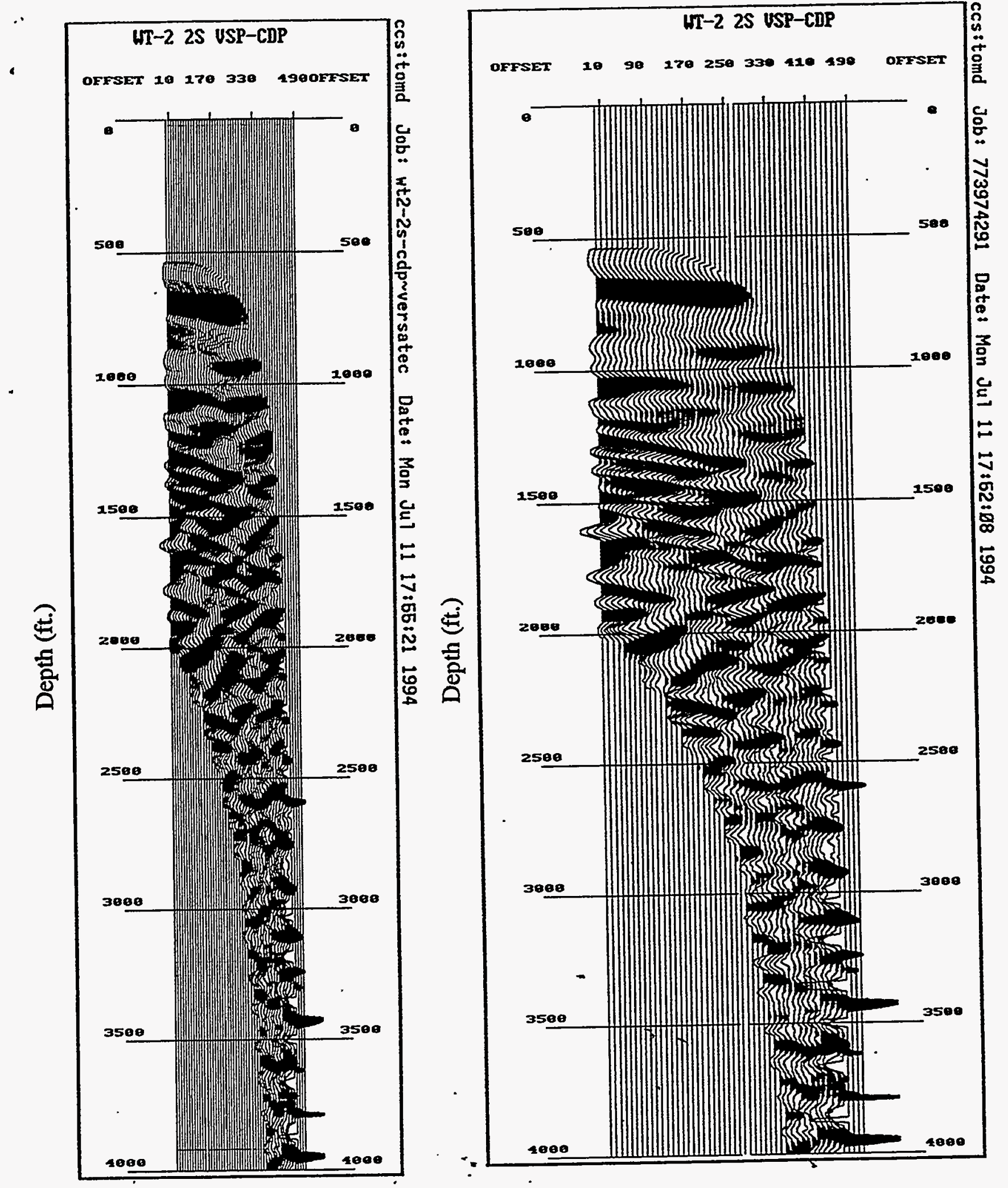

Figure 10a (left) VSP-CDP mapping of offset 2 S-wave source at 1:6000 scale.

Figure $10 \mathrm{~b}$ (right) VSP-CDP mapping of offset 2 S-wave source at 2 to 1 horizontal exaggeration. Offset labeled in feet from well. 


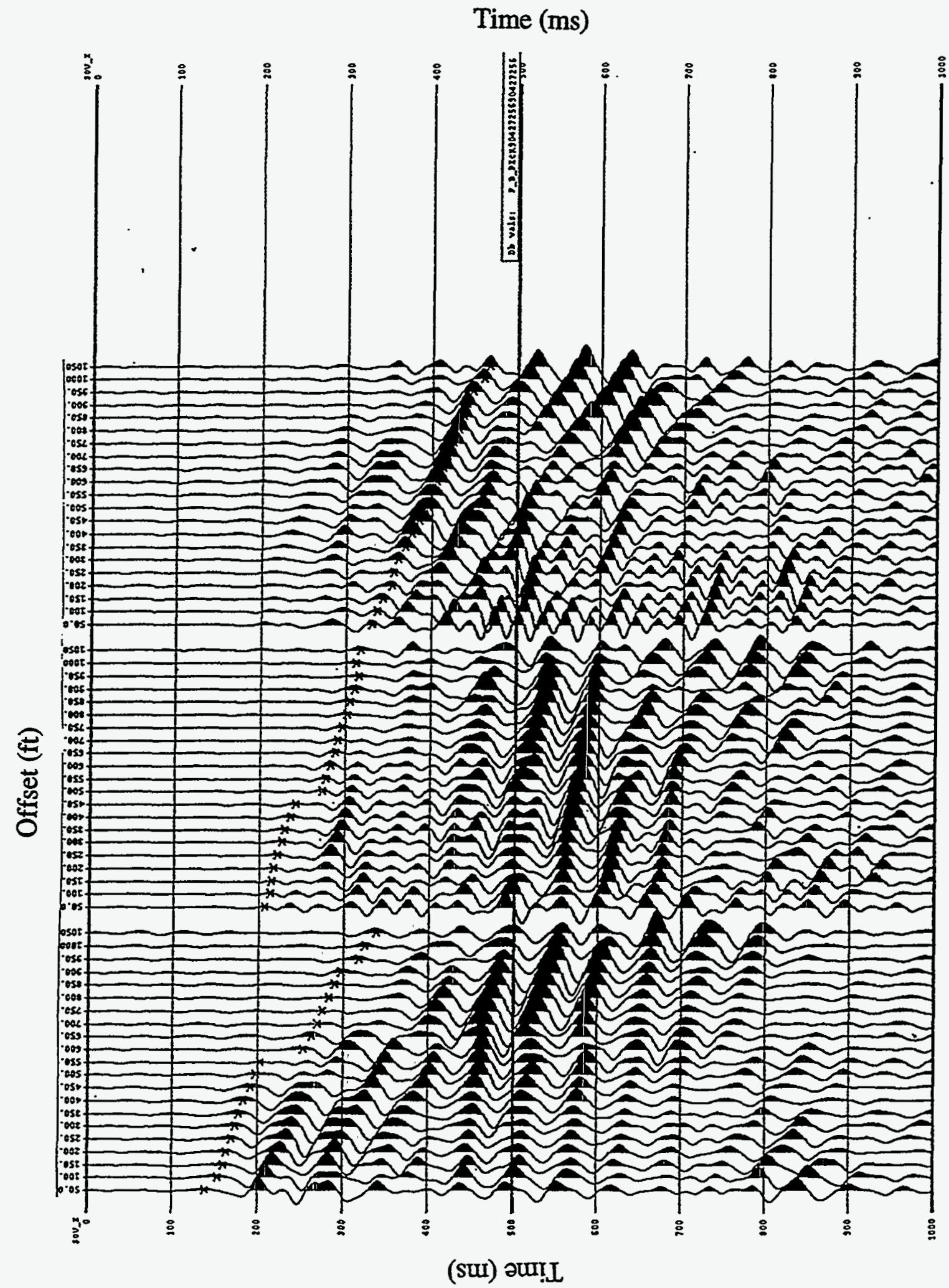

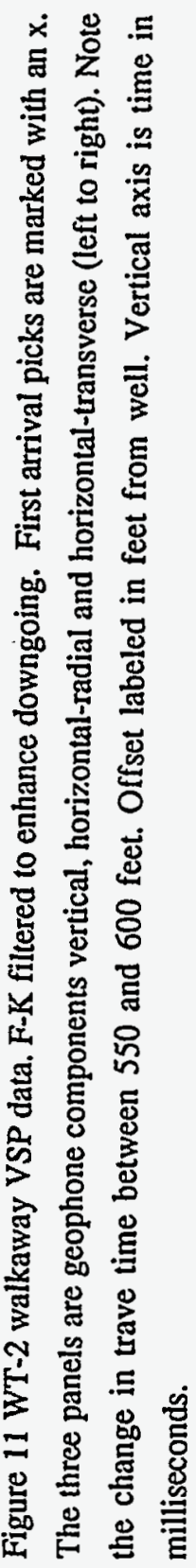


7

ccs:tomd Job: wt2-wkawy-cdp.versatec. Date: Sun Ju1 10 14:52:14 1994

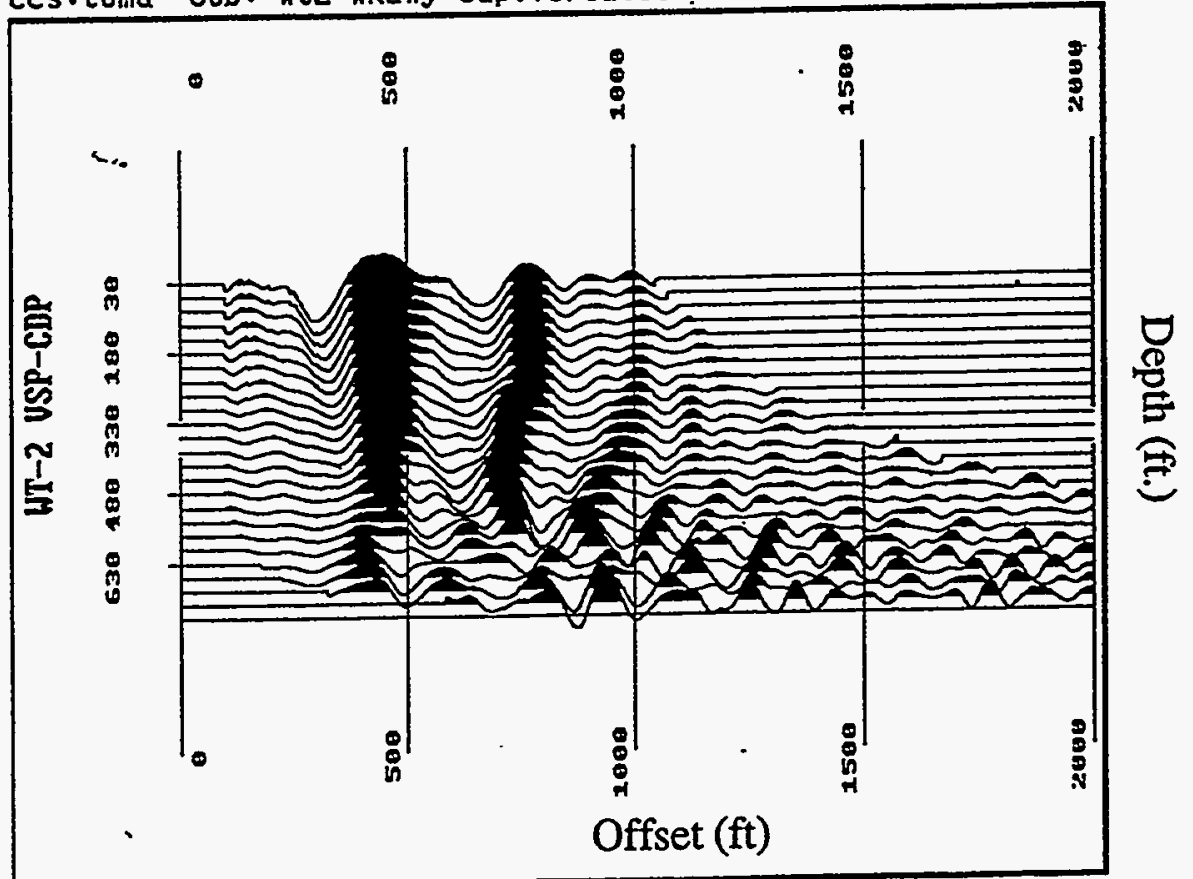

Figure 12 WT-2 walkaway VSP-CDP mapping. Horizontal axis is offset distance from 0 to 2000 feet, vertical axis from 30 to 750 feet is depth. Scale is 1:6000 for overlay on line 1. 


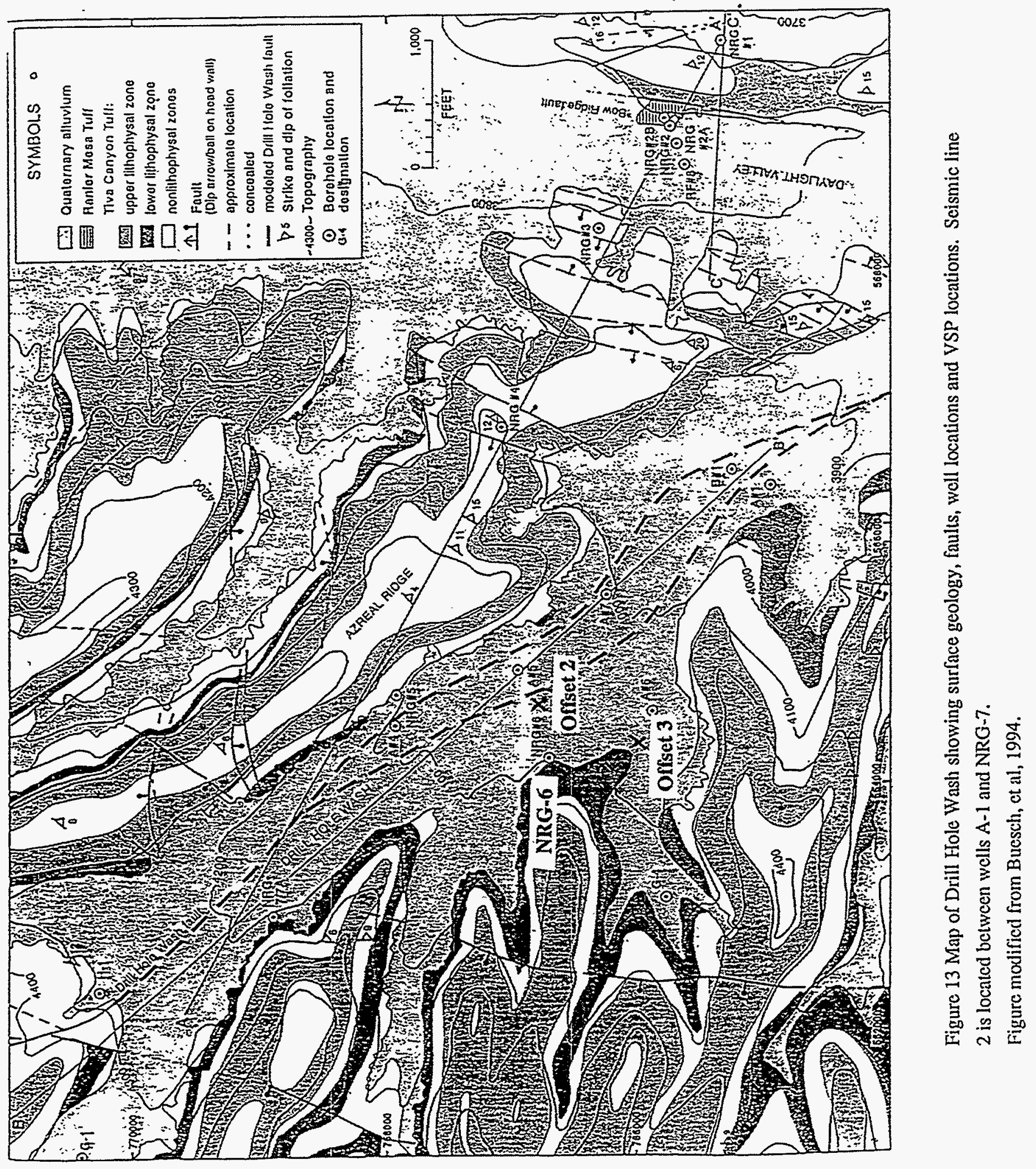




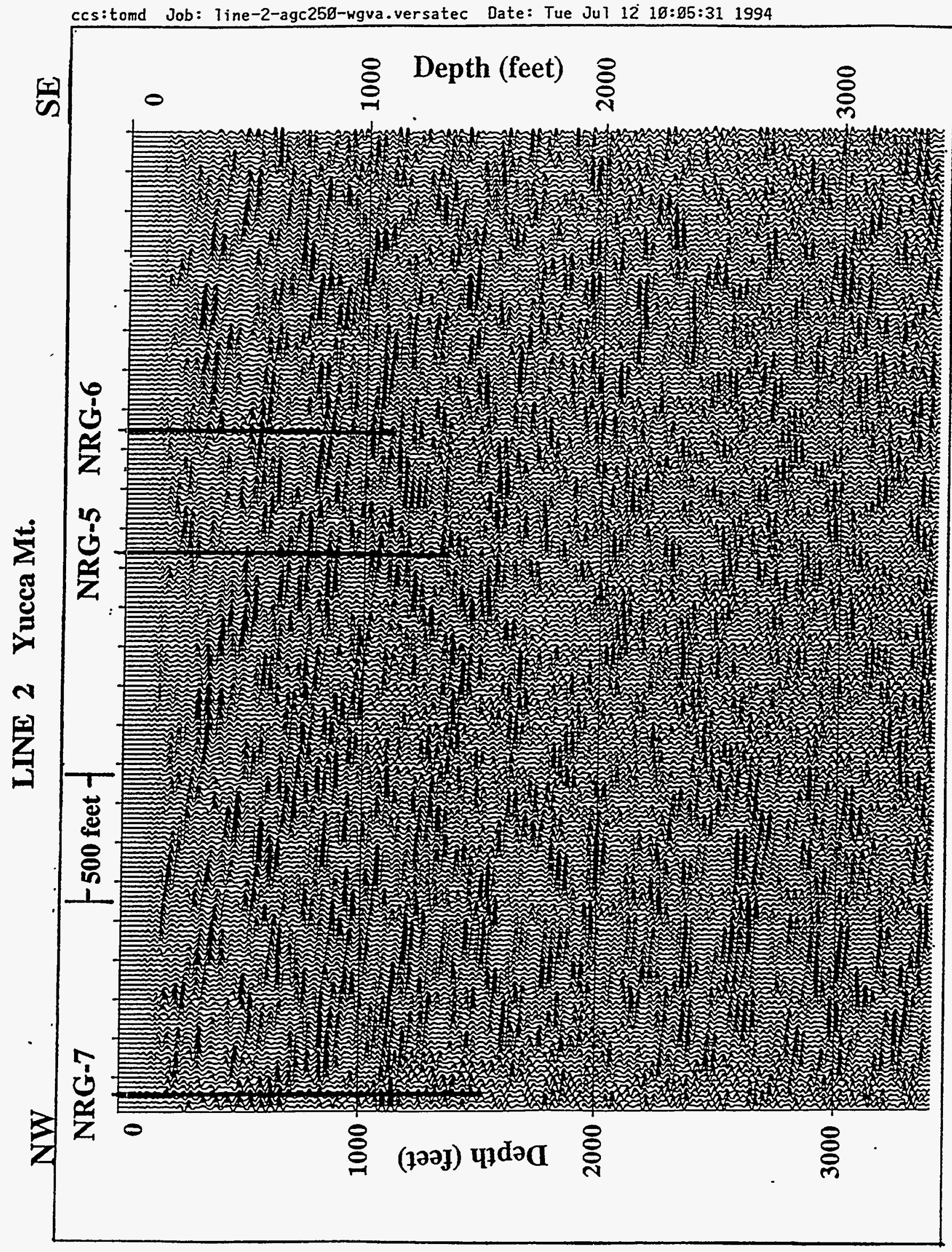

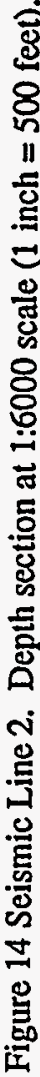




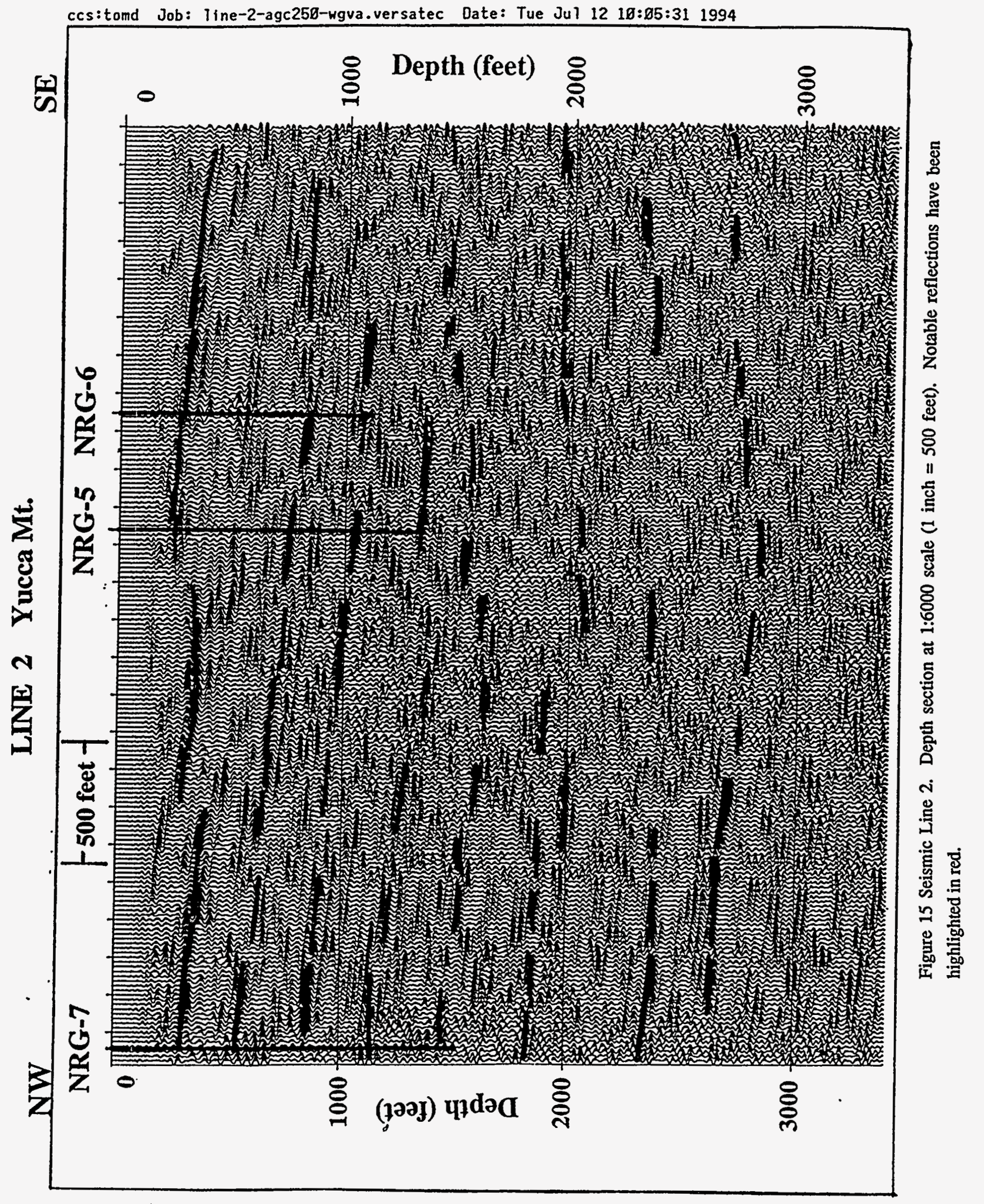


ccs:tomd Job: 1ine-2-agc25ஜ̈-wgva.versatec Date: Tue Jul 12 18:05:31 1994

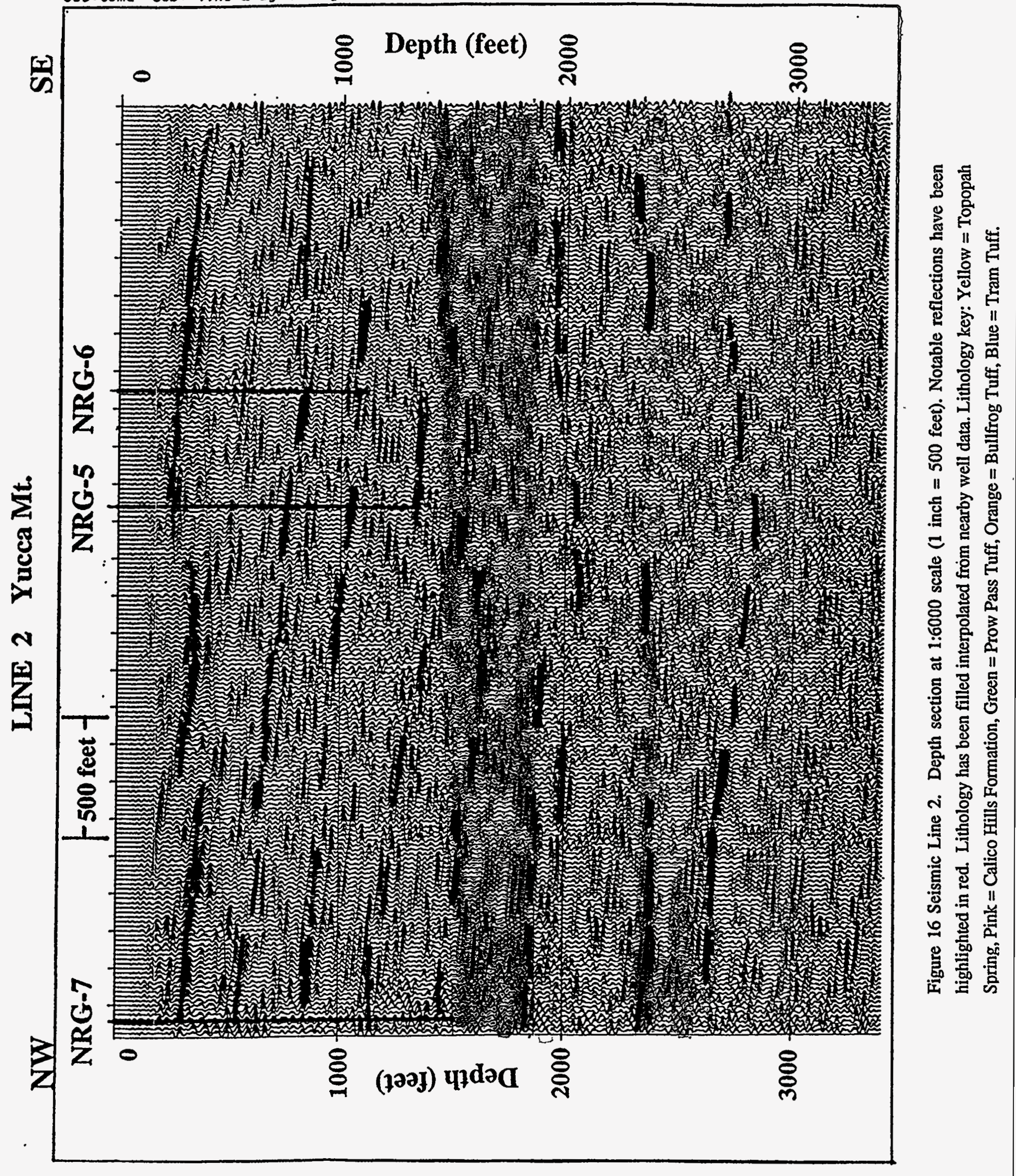




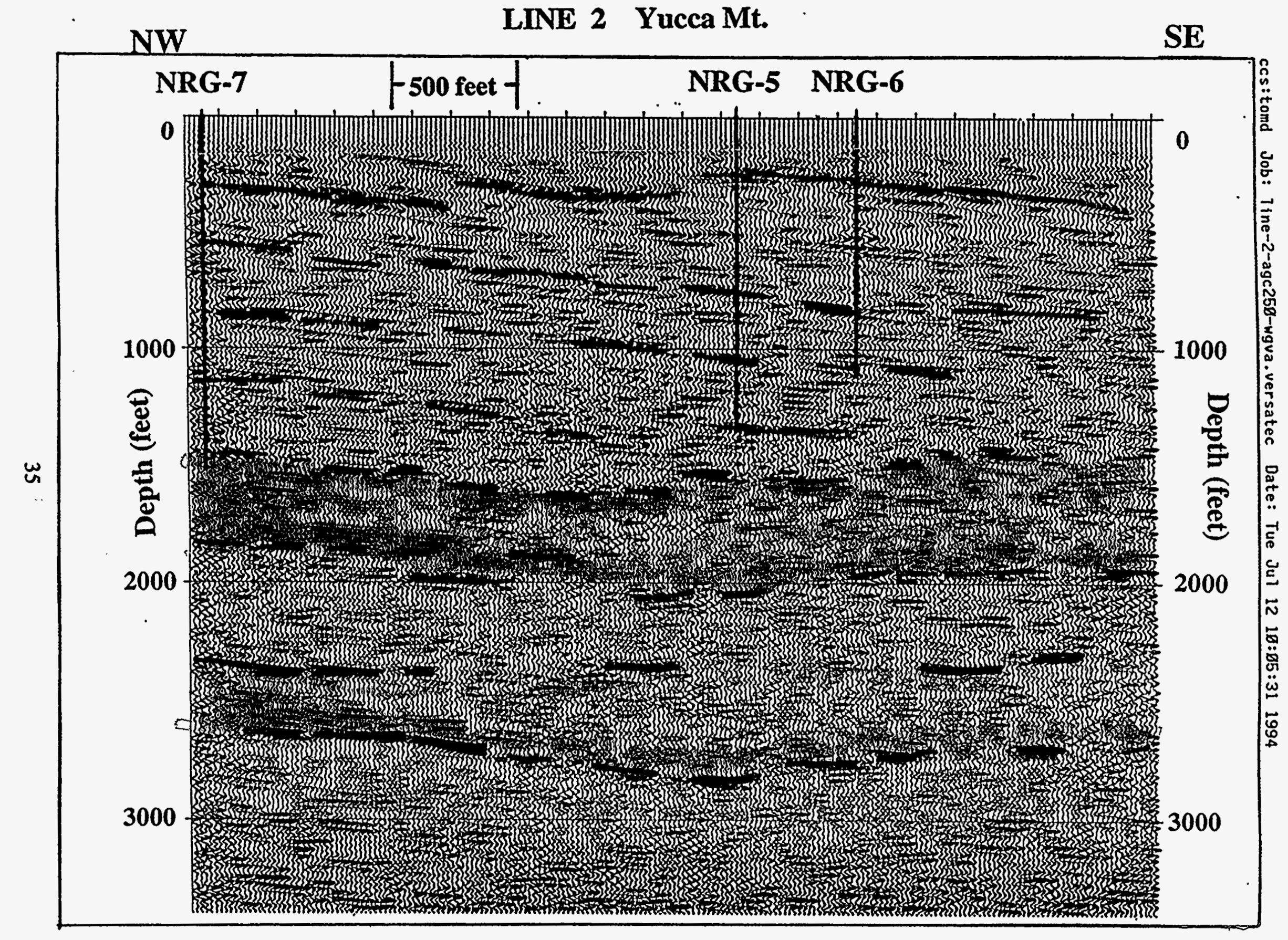

Figure 17 Seismic Line 2. Depth section at 1:6000 scale ( 1 inch $=500$ feet). Notable reflections have been highlighted in red. Lithology has been adjusted to seismic structure. Lithology key: Yellow = Topopah Spring, Pink $=$ Calico Hills Formation, Green $=$ Prow Pass Tuff, Orange $=$ Bullfrog Tuff, Blue $=$ Tram Tuff. 

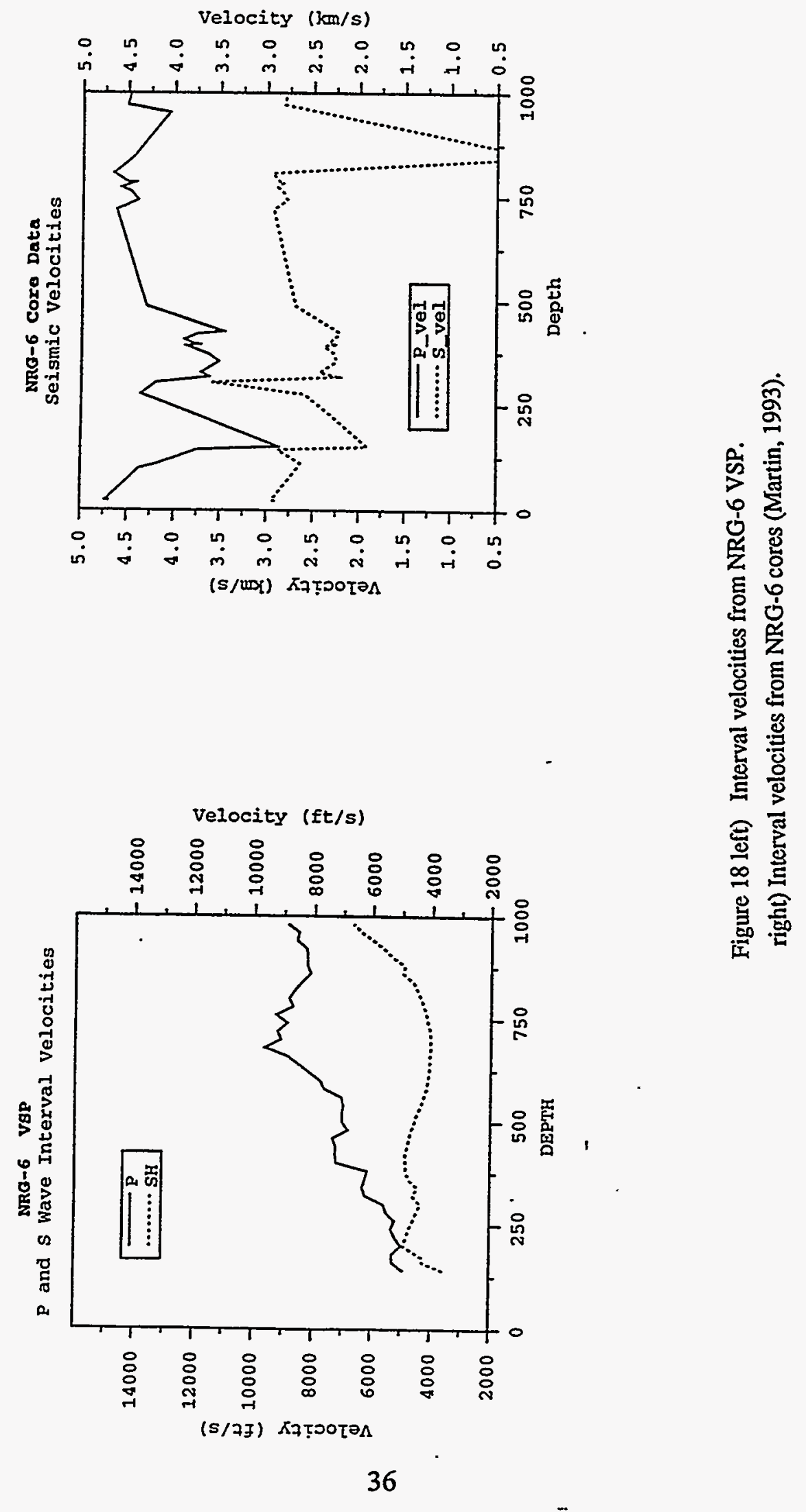


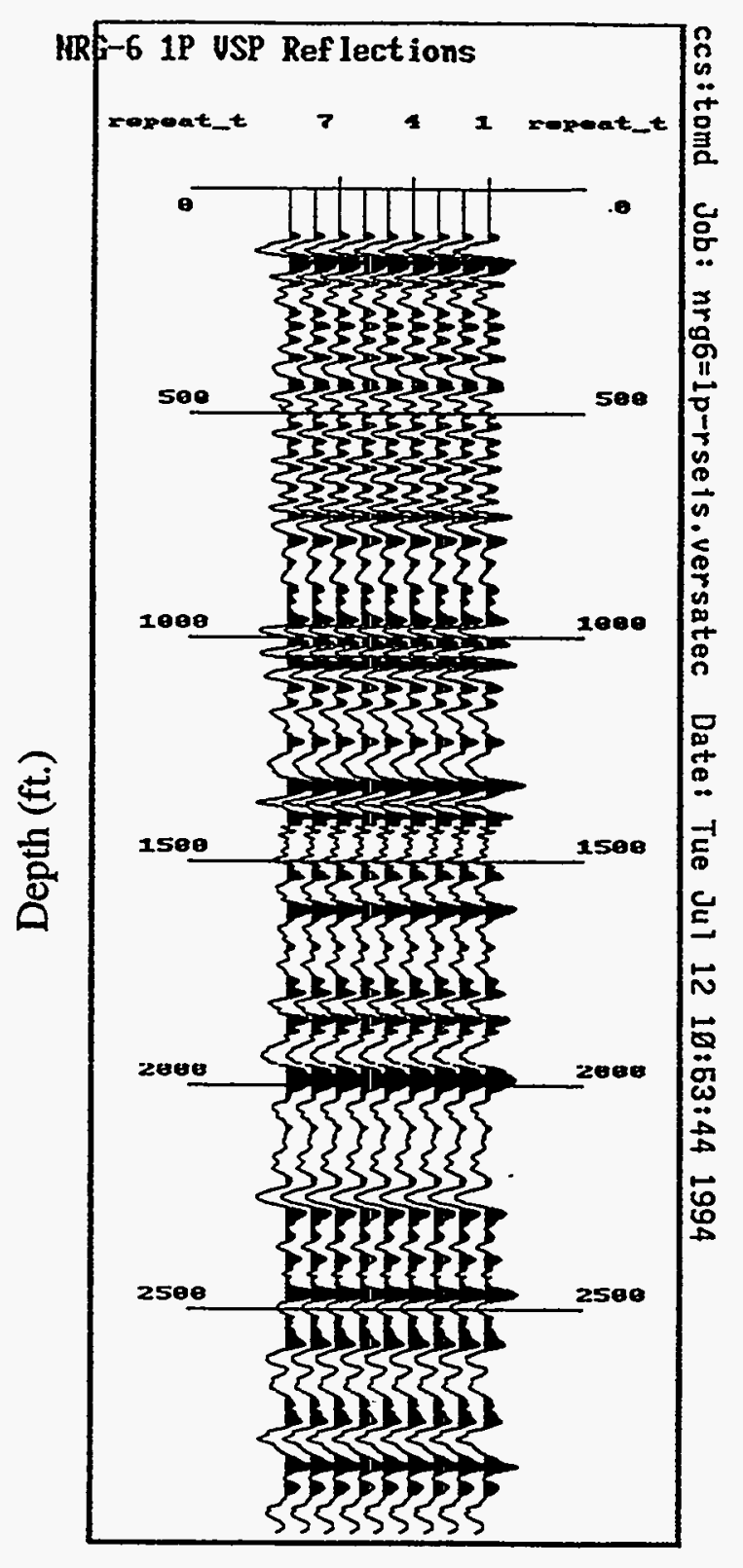

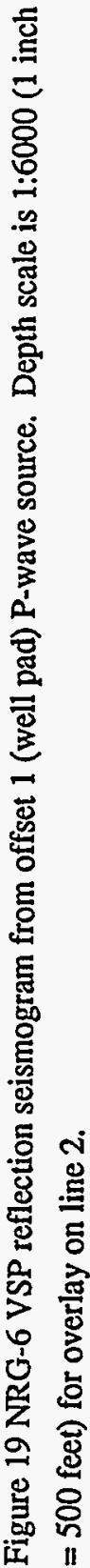




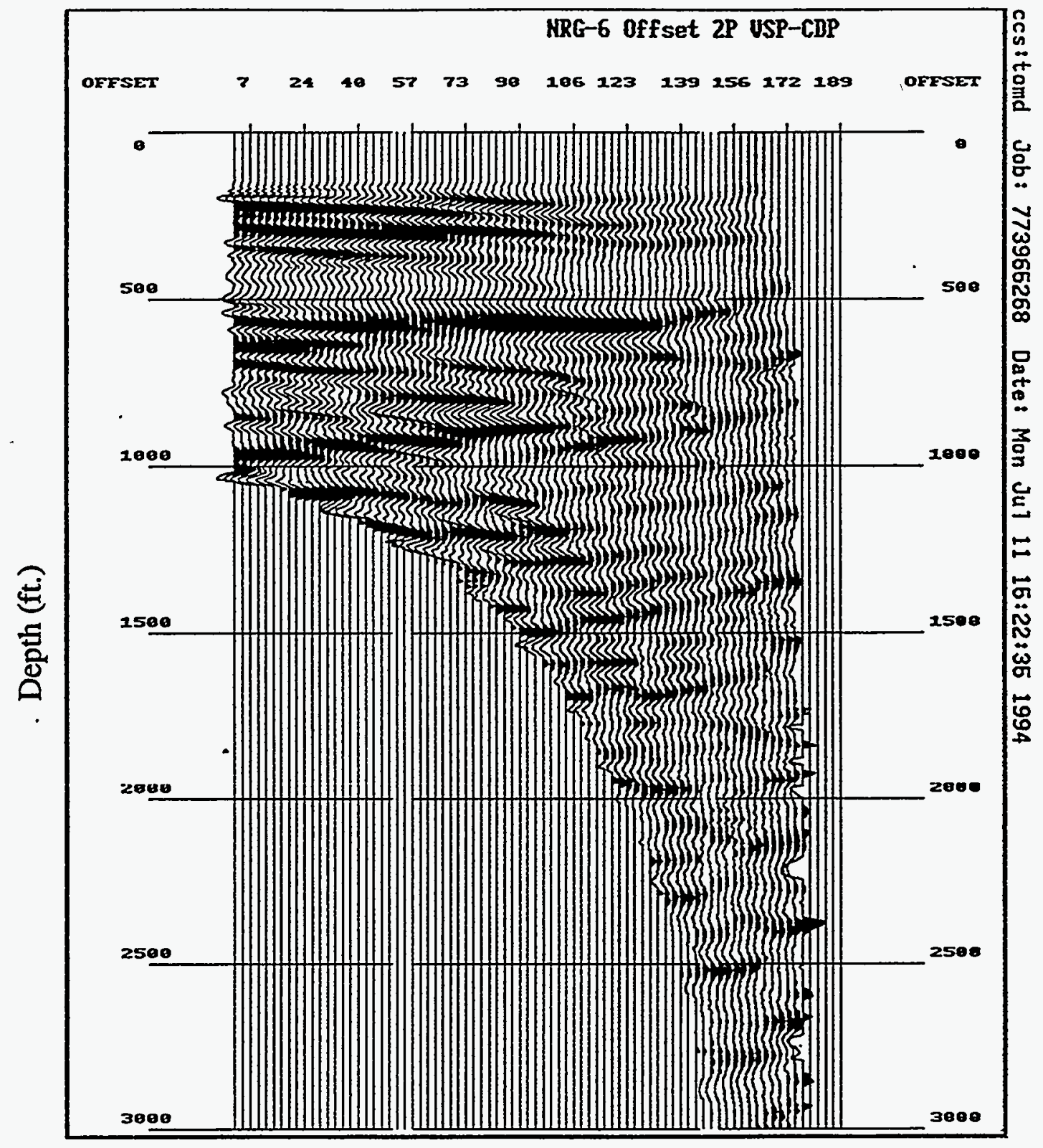

Figure 20a NRG-6 offset 2 P-wave source VSP-CDP mapping. Offset labeled in feet from well. Vertical axis is depth in feet. 


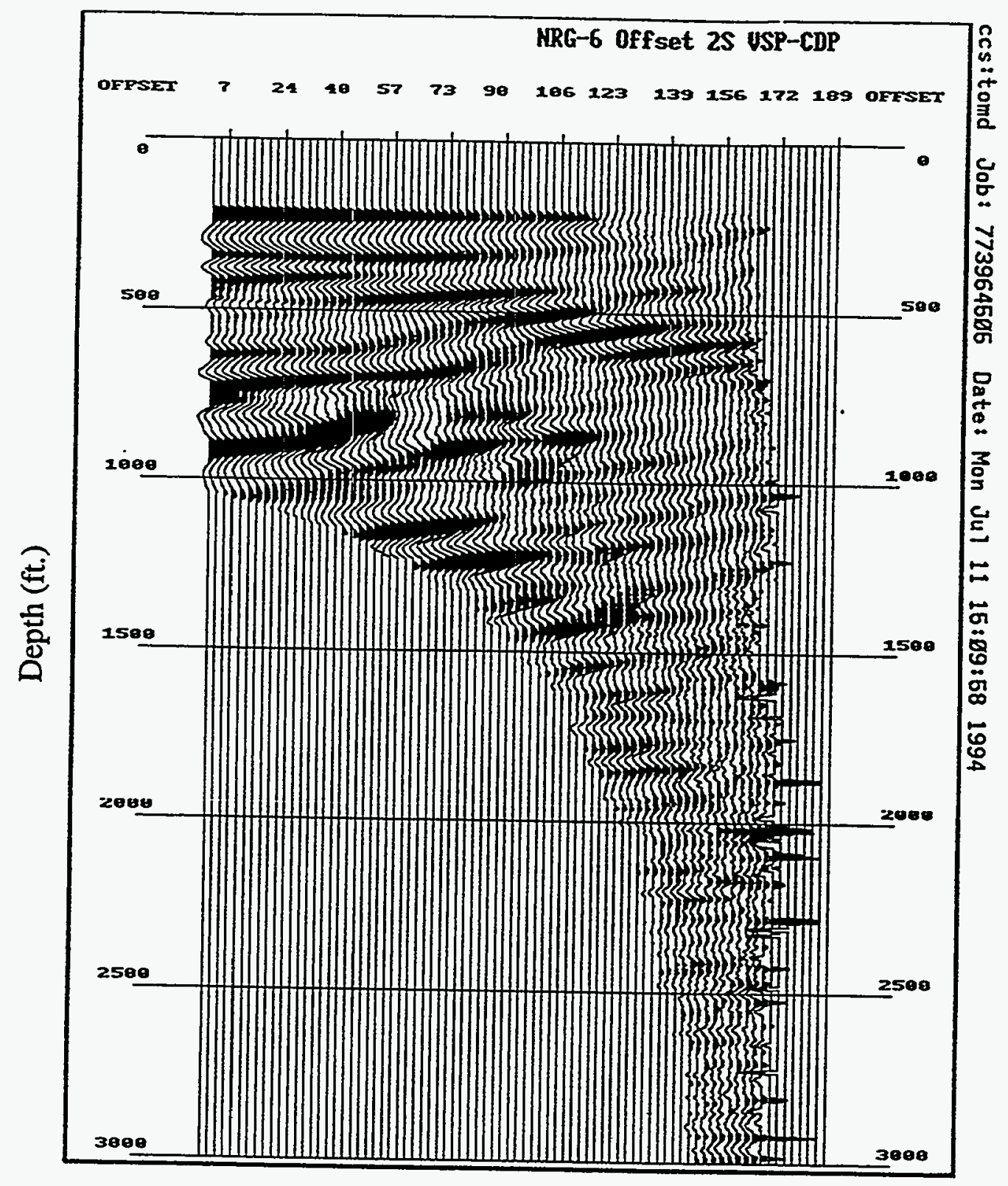

Figure 20b NRG-6 offset 2 S-wave source VSP-CDP mapping. Offset labeled in feet from well. Vertical axis is depth in feet. 
ccs:tomd Job: 773967259 Date: Mon JuT 11 15:59:15 1994

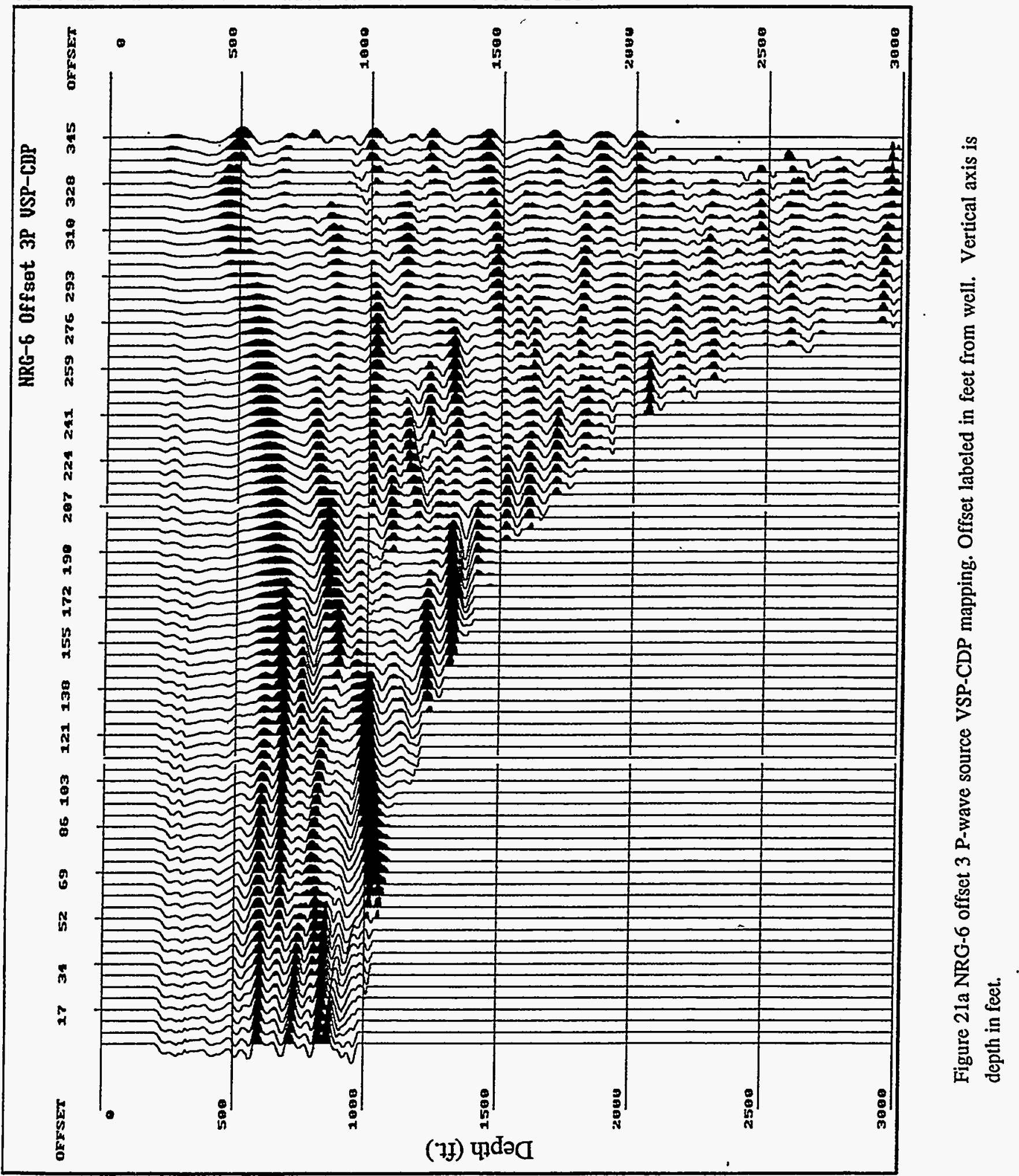


ccs:tomd Job: 773971254 Date: Mon Jul 11 17:02:57 1994

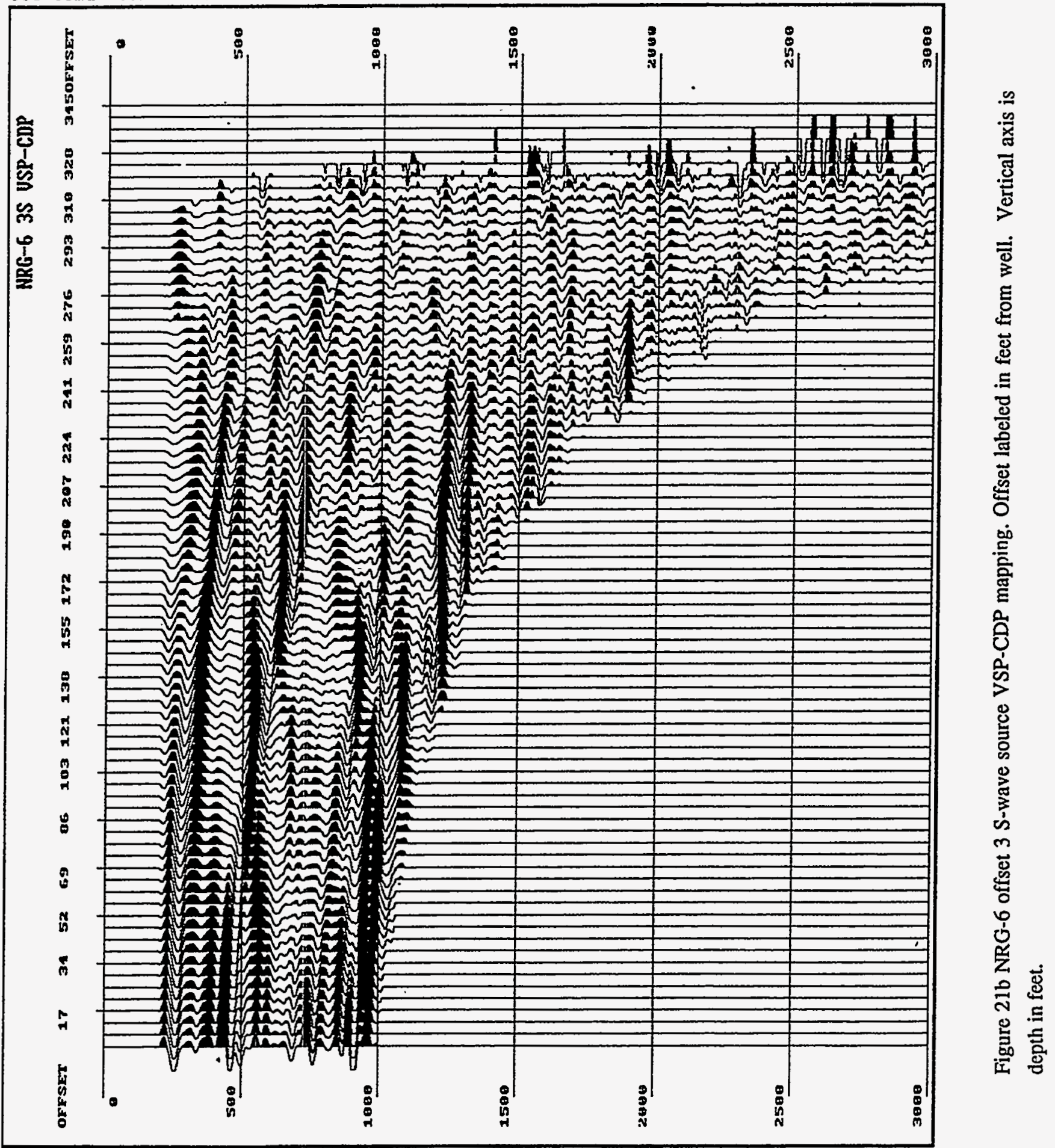

(अ) पाdəव 

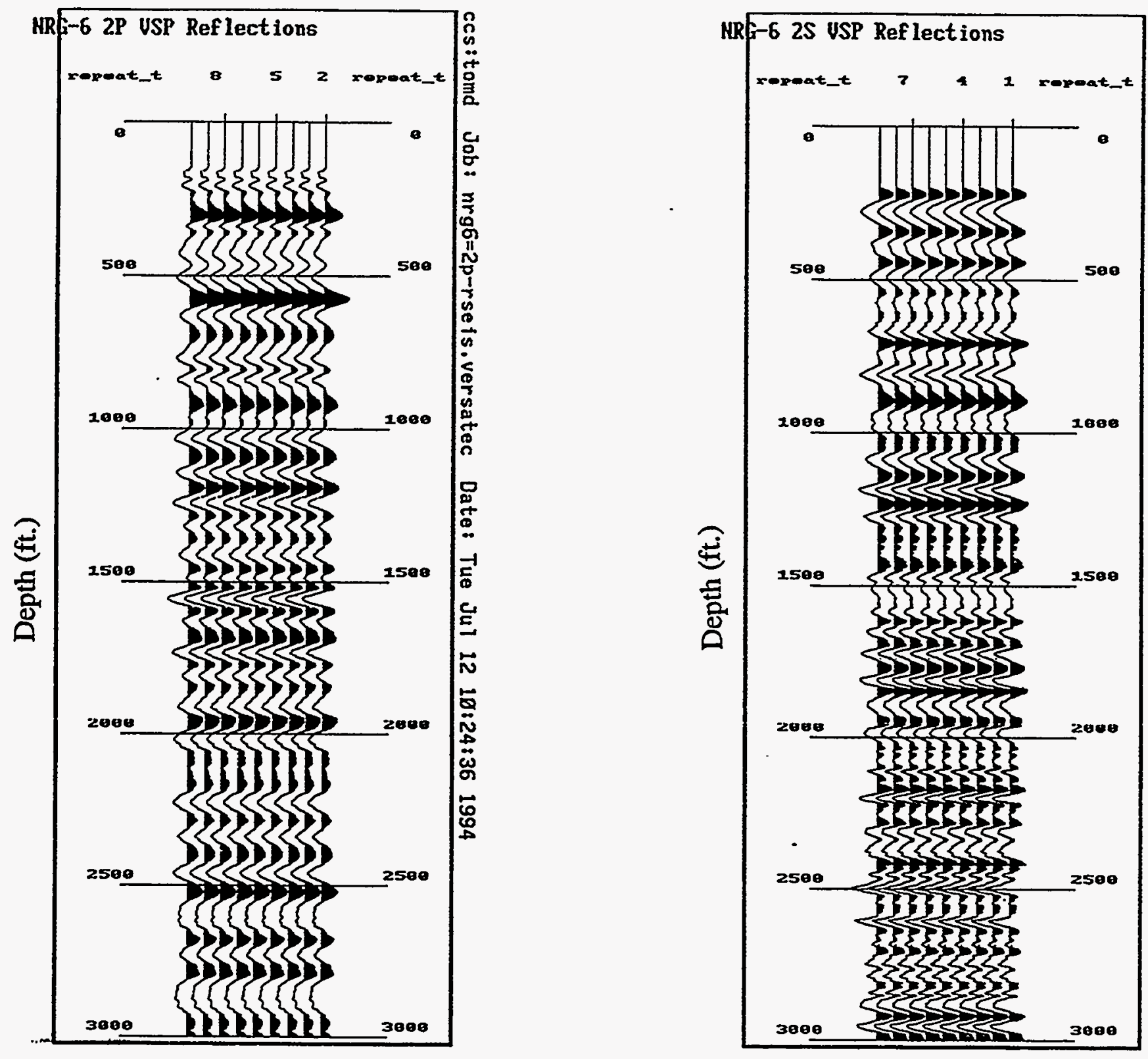

Figure 22a (left) NRG-6 offset 2 P-wave source reflection seismogram. Depth scale is $1: 6000(1$ inch $=500$ feet $)$ for overlay on line 2 .

Figure $22 b$ (right) NRG-6 offset 2 S-wave source reflection seismogram. Depth scale is $1: 6000(1$ inch $=500$ feet $)$ for overlay on line 2 . 

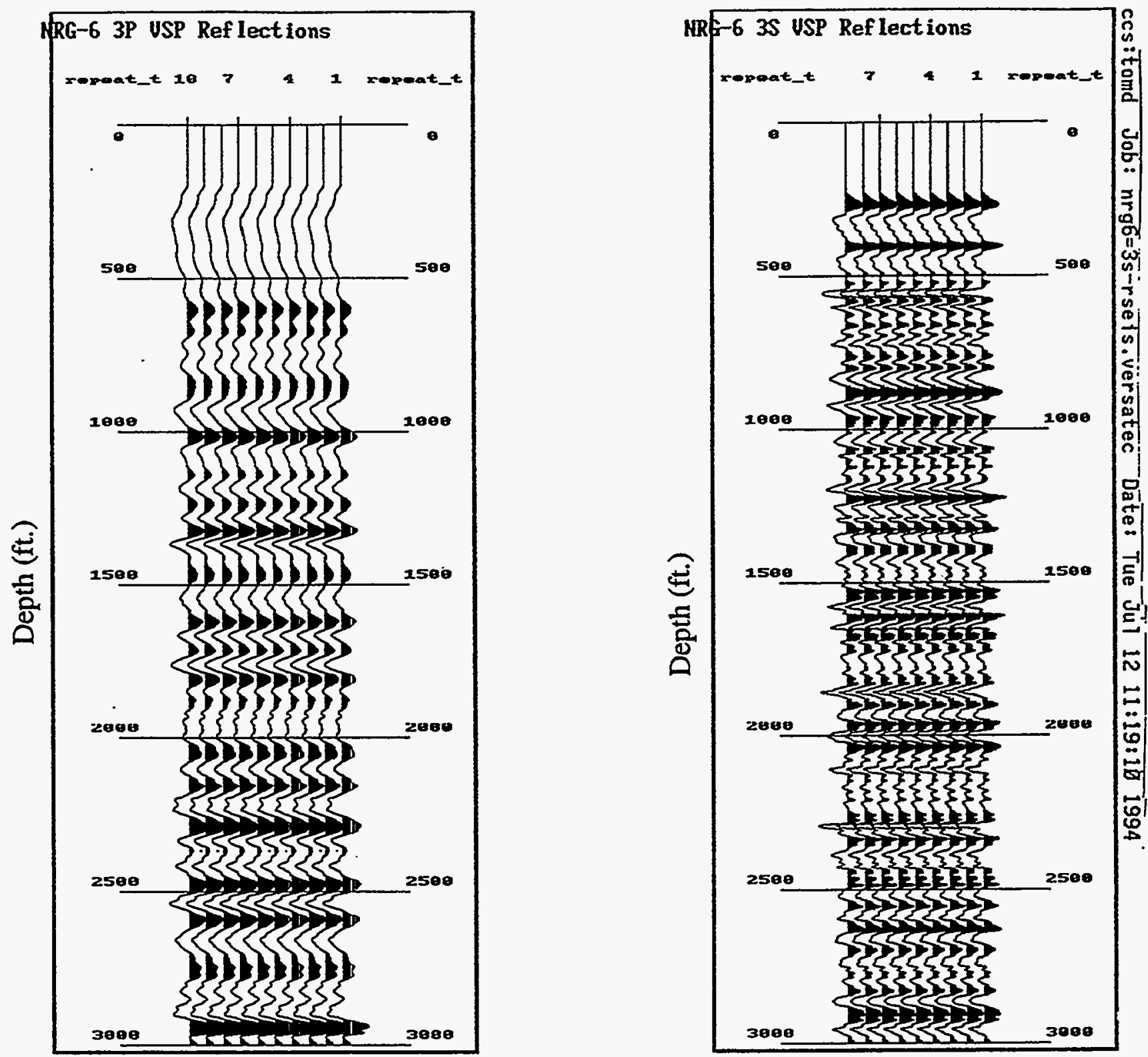

Figure 23a (left) NRG-6 offset 3 P-wave source reflection seismogram. Depth scale is $1: 6000$ ( 1 inch $=500$ feet) for overlay on line 2 .

Figure 23b (right) NRG-6 offset 3 S-wave source reflection seismogram. Depth scale is 1:6000 $(1 \mathrm{inch}=500$ feet) for overlay on line 2 . 


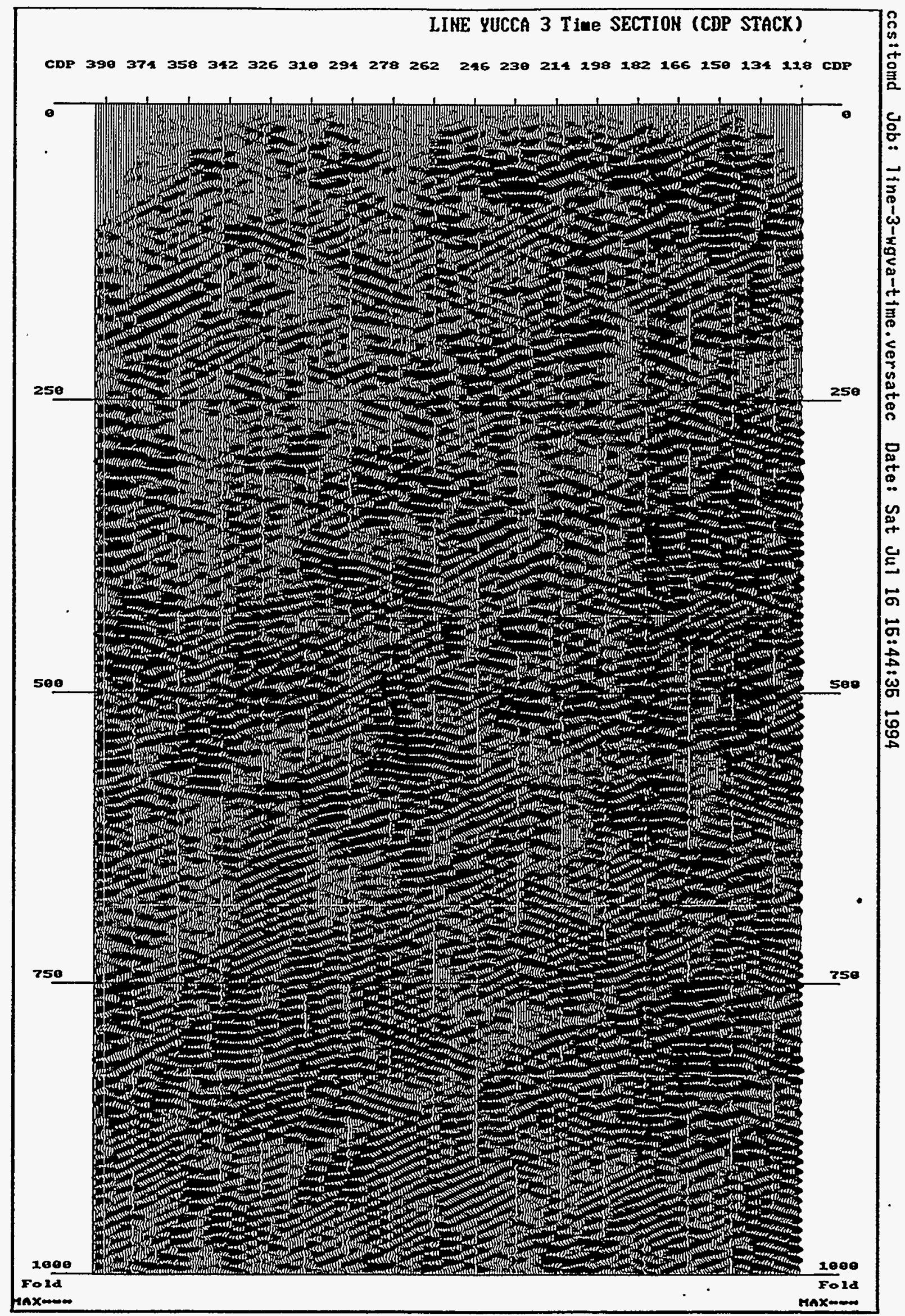

Figure 24 Seismic line 3 time section. Horizontal scale is 1:6000 ( 1 inch $=500$ feet). No interpretation is attempted because of data problems discussed in text. 